\title{
Time Delayed Stage-Structured Predator-Prey Model with Birth Pulse and Pest Control Tactics
}

\author{
Mei Yan, ${ }^{1,2}$ Yongfeng $\mathrm{Li}^{3}{ }^{3}$ and Zhongyi Xiang ${ }^{1,2}$ \\ ${ }^{1}$ Key Laboratory of Biologic Resources Protection and Utilization of Hubei Province, Enshi, Hubei 445000, China \\ ${ }^{2}$ Department of Mathematics, Hubei Minzu University, Enshi, Hubei 445000, China \\ ${ }^{3}$ Department of Mathematics and Information Sciences, Zhengzhou University of Light Industry, Zhengzhou, Henan 450002, China
}

Correspondence should be addressed to Zhongyi Xiang; yfxiang2007@163.com

Received 21 November 2013; Revised 16 January 2014; Accepted 18 January 2014; Published 7 April 2014

Academic Editor: Yanni Xiao

Copyright (c) 2014 Mei Yan et al. This is an open access article distributed under the Creative Commons Attribution License, which permits unrestricted use, distribution, and reproduction in any medium, provided the original work is properly cited.

\begin{abstract}
Normally, chemical pesticides kill not only pests but also their natural enemies. In order to better control the pests, two-time delayed stage-structured predator-prey models with birth pulse and pest control tactics are proposed and analyzed by using impulsive differential equations in present work. The stability threshold conditions for the mature prey-eradication periodic solutions of two models are derived, respectively. The effects of key parameters including killing efficiency rate, pulse period, the maximum birth effort per unit of time of natural enemy, and maturation time of prey on the threshold values are discussed in more detail. By comparing the two threshold values of mature prey-extinction, we provide the fact that the second control tactic is more effective than the first control method.
\end{abstract}

\section{Introduction}

The outbreak of pest often triggers serious ecological and economic problems. In recent years, the management of pest has increasingly become the focus of attention. How to effectively control pest is one primarily concern problem. In practice, lots of factors can affect the efficiency of pest control, for instance, the time of impulsive effects, the number of prey stocked or naturally released, and the proportion of killing or catching pests. Mathematical modelling is one of the main ways for estimating and predicting the range of ecological interactions between pest and predator. Lately, many papers have been devoted to propose and analyze the predator-prey systems [1-4].

In one aspect, many species have the life history that goes through two stages, immature and mature, which has significant morphological and behavioral differences between them. Therefore, it is necessary to account for these differences, and the dynamics of mathematical models with stage-structured prey-predator model has been widely studied [5-11] in recent years. In other aspects, in the natural world, the immature predator always undergo a certain time $\tau$ (which is called maturation time delay) to be mature, so time delays play an important role in biological meanings in age-structured models. Time delay and stage-structured systems are introduced into predator-prey models, which greatly enriches biologic background. Many age-structured models with time delay were extensively studied [12-14]. A single species model with stage structure and time delay was invested by Aiello and Freedman [15].

As far as the population dynamics is concerned, most models often considered that the population reproduces throughout all year. However, many species give birth seasonally or in regular pules. In this regard, the continuous reproduction of mature species should be removed from the model and termed this growth form as birth pulse. For instance, Tang and Chen study an age-structured model with density-dependent birth pulse in [16] and Xiang et al. [17] have considered a delayed Lotka-Volterra model with birth pulse and impulsive effect at different moment on the prey. Theories of impulsive differential equations have been studied lately [18-20]. Impulsive systems are found in many domains of applied sciences. The application of impulsive differential equations to population dynamics is an interesting topic since it is reasonable and corrects in modeling the evolution of populations. Virtually, the pulses occur at different moments 
more realistic, which is more meaningful than the pulses that occur at the same time. Recently, the qualitative analysis of impulsive differential equations at different moments has been widely investigated in many works [21-23].

Although, many authors have devoted to study the effects of pesticide on pest and its natural enemies and lots of instructive control strategies also have been given. An optimal time of pesticide applications still seems to be a novel interesting area. Following the practical pest management, we firstly propose the predator-prey model with pulse at the same time. Further, we assumed that the birth pulse and pest control tactics occur at different time. Discussing and comparing the mature prey-extinction of the two models, we get some new effective pest management.

\section{Predator-Prey Model with Impulsive Effects at the Same Time}

The purpose of this paper is to address how the time of impulsive effects influences the pest control. On the basis of the above discussion and motivated by $[8,16]$, we first will extend the following impulsive model which introduces the impulsive control tactics at the same time:

$$
\begin{gathered}
\dot{x}_{1}(t)=r x_{2}(t)-r e^{-\omega \tau} x_{2}(t-\tau)-\omega x_{1}(t), \\
\dot{x}_{2}(t)=r e^{-\omega \tau} x_{2}(t-\tau)-\beta x_{2}(t) y(t) \\
-d_{1} x_{2}(t)-d_{2} x_{2}^{2}(t), \quad t \neq n T, \\
\dot{y}(t)=k \beta x_{2}(t) y(t)-d_{3} y(t), \\
x_{1}\left(t^{+}\right)=\left(1-p_{0}\right) x_{1}(t)+\mu, \\
x_{2}\left(t^{+}\right)=x_{2}(t), \quad b y(t) \\
y\left(t^{+}\right)=(1-p) y(t)+\frac{b y}{a+y(t)}, \\
\left(\omega_{1}(s), \omega_{2}(s), \omega_{3}(s)\right) \in C_{+} \quad \omega_{i}(0)>0, i=1,2,3, \\
=C\left([-\tau, 0], R_{+}^{3}\right),
\end{gathered}
$$

where $x_{1}(t)$ and $x_{2}(t)$ are densities of the immature prey and mature prey, respectively. $y(t)$ denotes the density of predator. $\tau$ represents a constant time to maturity and $\omega, d_{1}, d_{2}, d_{3}, k, a, b, \beta, r$ are positive constants. $r$ is the birth rate of immature prey, $\omega\left(\omega>d_{3}\right), d_{1}$ are the mortality rates of the immature prey and mature prey, and $d_{3}\left(d_{3}>d_{1}\right)$ is the death coefficient of $y(t) . d_{2}$ is the intraspecific competition rate of the mature prey. $\beta$ is the transmission coefficient. The term $r e^{-\omega \tau} x_{2}(t-\tau)$ represents the immatures who were born at time $t-\tau$ and survive at time $t$ (with the immature death rate $\omega$ ) and therefore represents the transformation of immatures to matures $\left(r e^{-\omega \tau}>d_{1}\right)$. $k$ is the rate of conversing prey into predator. $p_{0}, \quad p\left(0 \leq p_{0}, p<1\right)$ represent the killing (or poisoning) rate of the prey and the predator, respectively. $\mu \geq 0$ is the stocking amount of the immature prey at $t=n T$. $b y(t) /(a+y(t))$ denotes the birth effort of predator population at $t=n T$, where $b$ means the maximum birth effort per unit of time and $a$ the predator size at which $50 \%$ saturation $\left(b y(t) /\left.(a+y(t))\right|_{y(t)=a}=b / 2\right)$ measures how soon saturation occurs.
Lemma 1 (see [24]). Consider the following equation:

$$
\dot{x}(t)=a x(t-\tau)-b x(t)-c x^{2}(t),
$$

where $a, b, c, \tau>0$ for $-\tau \leq t \leq 0$. We have

(i) if $a<b$, then $\lim _{t \rightarrow \infty} x(t)=0$;

(ii) if $a>b$, then $\lim _{t \rightarrow \infty} x(t)=(a-b / c)$.

Lemma 2. Consider the following system:

$$
\begin{array}{r}
\dot{u}(t)=-d_{3} u(t), \quad t \neq n T, \\
u\left(t^{+}\right)=(1-p) u(t)+\frac{b u(t)}{a+u(t)}, \quad t=n T,
\end{array}
$$

(i) if $p>1+b / a-e^{d_{3} T}$, the fixed point $u_{0}^{*}=0$ is globally asymptotically stable;

(ii) if $p<1+b / a-e^{d_{3} T}$, the fixed point $u^{*}=(a(1-p)+$ $\left.b-a e^{d_{3} T}\right) /\left(1-(1-p) e^{-d_{3} T}\right)$ is globally asymptotically stable.

Proof. Integrating the first equation of (3) on $n T<t<(n+$ 1) $T$, we have

$$
u(t)=u\left(n T^{+}\right) e^{-d_{3}(t-n T)}, \quad n T \leq t \leq(n+1) T .
$$

With the successive pulse, we can obtain the following stroboscopic map of system (3):

$$
\begin{aligned}
u\left((n+1) T^{+}\right)= & (1-p) u\left(n T^{+}\right) e^{-d_{3} T} \\
& +\frac{b u\left(n T^{+}\right) e^{-d_{3} T}}{a+u\left(n T^{+}\right) e^{-d_{3} T}}
\end{aligned}
$$

Let $F(u)=(1-p) u e^{-d_{3} T}+\left(b u e^{-d_{3} T}\right) /\left(a+u e^{-d_{3} T}\right)$. If $p>$ $1+(b / a)-e^{d_{3} T}$, then the fixed point $u_{0}^{*}=0$, since

$$
\left|\frac{d F(u)}{d u}\right|_{u=0}=(1-p) e^{-d_{3} T}+\frac{b e^{-d_{3} T}}{a}<1 ;
$$

and unstable if $p<1+(b / a)-e^{d_{3} T}$, since

$$
\left|\frac{d F(u)}{d u}\right|_{u=0}=(1-p) e^{-d_{3} T}+\frac{b e^{-d_{3} T}}{a}>1 .
$$

If $p<1+(b / a)-e^{d_{3} T}$, (5) has a unique positive fixed point:

$$
u^{*}=\frac{a(1-p)+b-a e^{d_{3} T}}{1-(1-p) e^{-d_{3} T}} .
$$

By the same way, we can obtain the fact that the fixed point $u^{*}=\left(a(1-p)+b-a e^{d_{3} T}\right) /\left(1-(1-p) e^{-d_{3} T}\right)$ is globally asymptotically stable if $p<1+(b / a)-e^{d_{3} T}$. This completes the proof. 
which implies that corresponding periodic solution of (3) on $(n T,(n+1) T]$ is

$$
\widetilde{u}(t)=\frac{a(1-p)+b-a e^{d_{3} T}}{1-(1-p) e^{-d_{3} T}} e^{-d_{3}(t-n T)} .
$$

Thus (1) has a mature prey-extinction periodic solution $\left(\tilde{x}_{1}(t), 0, \tilde{y}(t)\right)$, where $\tilde{x}_{1}(t)=\left(\mu e^{-\omega(t-n T)}\right) /(1-(1-$ $\left.\left.p_{0}\right) e^{-\omega T}\right), \tilde{y}(t)=\left(a(1-p)+b-a e^{d_{3} T}\right) /(1-(1-$ p) $\left.e^{-d_{3} T}\right) e^{-d_{3}(t-n T)}$.

Theorem 3. The mature prey-extinction periodic solution $\left(\tilde{x}_{1}(t), 0, \tilde{y}(t)\right)$ is globally asymptotically stable if

$$
R_{1}<1
$$

holds, where $R_{1}=\left(r e^{-\omega \tau}-d_{1}\right)\left[1-(1-p) e^{-d_{3} T}\right] / \beta[a(1-$ p) $\left.e^{-d_{3} T}+b e^{-d_{3} T}-a\right]$.

Proof. Let $\left(x_{1}(t), x_{2}(t), y(t)\right)$ be any solution of system (1). Following from the third equation of system (1), we notice that $\dot{y}(t) \geq-d_{3} y(t)$. Consider the following impulsive differential system:

$$
\begin{gathered}
\dot{y}_{1}(t)=-d_{3} y_{1}(t), \quad t \neq n T, \\
y_{1}\left(t^{+}\right)=(1-p) y_{1}(t)+\frac{b y_{1}(t)}{a+y_{1}(t)}, \quad t=n T .
\end{gathered}
$$

According to Lemma 2, we obtain the fact that $\tilde{y}_{1}(t)=((a(1-$ $\left.\left.p)+b-a e^{d_{3} T}\right) /\left(1-(1-p) e^{-d_{3} T}\right)\right) e^{-d_{3}(t-n T)}$, for $n T<t \leq(n+1) T$. By using the comparison differential theorem of impulsive equation, we have $y(t) \geq y_{1}(t)$ and $y_{1}(t) \rightarrow \tilde{y}_{1}(t)$ as $t \rightarrow \infty$. Therefore, for an arbitrarily small positive constant $\varepsilon$ and all $t$ large enough:

$$
y(t) \geq y_{1}(t) \geq \tilde{y}_{1}(t)-\varepsilon
$$

holds true.

For simplicity, we assume that (12) holds for all $t>0$. From (12), we have

$$
\begin{aligned}
\frac{d x_{2}(t)}{d t} \leq & r e^{-\omega \tau} x_{2}(t-\tau)-\left(\beta\left(\tilde{y}_{1}(t)-\varepsilon\right)+d_{1}\right) x_{2}(t) \\
& -d_{2} x_{2}^{2}(t), \quad t>n T+\tau .
\end{aligned}
$$

Consider the following comparison differential equation:

$$
\begin{aligned}
\frac{d x(t)}{d t}= & r e^{-\omega \tau} x(t-\tau) \\
& -\left(\beta\left(\tilde{y}_{1}(t)-\varepsilon\right)+d_{1}\right) x(t)-d_{2} x^{2}(t), \\
t & >n T+\tau .
\end{aligned}
$$

From (10) and Lemma 1, we have $\lim _{t \rightarrow \infty} x(t)=0$. By the comparison differential theorem, we get $\lim _{t \rightarrow \infty} x_{2}(t)<$ $\lim _{t \rightarrow \infty} x(t)=0$. Noticing the positivity of $x_{2}(t)$, we know that $\lim _{t \rightarrow \infty} x_{2}(t)=0$. Without loss of generality, we assume that for any $\varepsilon_{1}$ (sufficiently small)

$$
x_{2}(t) \leq \varepsilon_{1} .
$$

Following from (1) and (15), we can obtain that

$$
-r e^{-\omega \tau} \varepsilon_{1}-\omega x_{1}(t) \leq \dot{x}_{1}(t) \leq r \varepsilon_{1}-\omega x_{1}(t),
$$

which yields $z_{1}(t) \leq x_{1}(t) \leq z_{2}(t)$ and $z_{1}(t) \rightarrow \tilde{x}_{1}(t)$, $z_{2}(t) \rightarrow \tilde{x}_{1}(t)$ as $t \rightarrow \infty \rightarrow \infty$, while $z_{1}(t), z_{2}(t)$ are the solutions of

$$
\begin{aligned}
& \dot{z}_{1}(t)=-r e^{-\omega \tau} \varepsilon_{1}-\omega z_{1}(t), \quad t \neq n T, \\
& \Delta z_{1}(t)=-p_{0} z_{1}(t)+\mu, \quad t=n T, n \in Z^{+}, \\
& \dot{z}_{2}(t)=r \varepsilon_{1}-\omega z_{2}(t), \quad t \neq n T, \\
& \Delta z_{2}(t)=-p_{0} z_{2}(t)+\mu, \quad t=n T, n \in Z^{+},
\end{aligned}
$$

respectively. Here, $\widetilde{z}_{1}(t)=-r e^{-\omega \tau} \varepsilon_{1} / \omega+((\mu+$ $\left.\left.\left(\left(r e^{-\omega \tau} \varepsilon_{1}\right) / \omega\right) p_{0}\right) /\left(1-\left(1-p_{0}\right) e^{-\omega T}\right)\right) e^{-\omega(t-n T)}$ and $\tilde{z}_{2}(t)=$ $\left(r \varepsilon_{1} / \omega\right)+\left(\left(\mu+\left(r \varepsilon_{1} / \omega\right) p_{0}\right) /\left(1-\left(1-p_{0}\right) e^{-\omega T}\right)\right) e^{-\omega(t-n T)}$, for $t \in(n T,(n+1) T]$.

In view of the comparison differential theorem, for any $\varepsilon_{2}$, let $\varepsilon_{1} \rightarrow 0$; then $\widetilde{z}_{1}(t)-\varepsilon_{2}<x_{1}(t)<\widetilde{z}_{2}(t)+\varepsilon_{2}$, for $t$ large enough, which means $x_{1}(t) \rightarrow \tilde{x}_{1}(t)$ as $t \rightarrow \infty$.

Next, we will prove that $y(t) \rightarrow \tilde{y}(t)$ as $t \rightarrow \infty$. From (1) and (15), we get

$$
-d_{3} y(t) \leq \dot{y}(t) \leq-\left(d_{3}-k \beta \varepsilon_{1}\right) y(t) .
$$

For the left hand inequality, it follows from impulsive differential equation (5) that $y(t) \geq y_{1}(t)$ and $y_{1}(t) \rightarrow \tilde{y}_{1}(t)$ as $t \rightarrow \infty$. For the right hand inequality, we consider the following impulsive differential equation:

$$
\begin{aligned}
& \dot{z}_{3}(t)=-\left(d_{3}-k \beta \varepsilon_{1}\right) z_{3}(t), \quad t \neq n T, \\
& z_{3}\left(t^{+}\right)=(1-p) z_{3}(t)+\frac{b z_{3}(t)}{a+z_{3}(t)}, \quad t=n T, n \in Z^{+},
\end{aligned}
$$

and $\widetilde{z}_{3}(t)=\left(\left(a(1-p)+b-a e^{d_{3} T}\right) /(1-(1-\right.$ p) $\left.\left.e^{-d_{3} T}\right)\right) e^{-\left(d_{3}-\lambda \beta \varepsilon_{1}\right)(t-n T)}$.

Therefore, for any $\varepsilon_{3}, \tilde{y}_{1}(t)-\varepsilon_{3}<y(t)<\widetilde{z}_{3}(t)+\varepsilon_{3}$. Let $\varepsilon_{1} \rightarrow 0$; then we get

$$
\tilde{y}(t)-\varepsilon_{3}<y(t)<\tilde{y}(t)+\varepsilon_{3}
$$

for $t$ large enough, which means $y(t) \rightarrow \tilde{y}(t)$ as $t \rightarrow \infty$. The proof is completed.

Corollary 4. (i) If $p<1+\left(b \beta /\left(\left(r e^{-\omega \tau}-d_{1}\right)+a \beta\right)\right)-$ $e^{d_{3} T}$ holds, then the mature prey-extinction periodic solution $\left(\tilde{x}_{1}(t), 0, \tilde{y}(t)\right)$ is globally asymptotically stable.

(ii) If $T<\left(1 / d_{3}\right) \ln \left(1+\left(b \beta /\left(\left(r e^{-\omega \tau}-d_{1}\right)+a \beta\right)\right)-\right.$ p) holds, then the mature prey-extinction periodic solution $\left(\tilde{x}_{1}(t), 0, \tilde{y}(t)\right)$ is globally asymptotically stable.

In biological terms, since $p<1+\left(b \beta /\left(\left(r e^{-\omega \tau}-d_{1}\right)+a \beta\right)\right)-$ $e^{d_{3} T}$ or $T<\left(1 / d_{3}\right) \ln \left(1+\left(b \beta /\left(\left(r e^{-\omega \tau}-d_{1}\right)+a \beta\right)\right)-p\right)$, the mature prey will extinct and immature prey and predators will coexist. 


\section{Predator-Prey Model with Impulsive Effects at the Different Time}

In particular, in order to avoid the adverse effects of pesticides on the newly released natural enemies, we consider the following method implemented in practice to avoid such antagonism. That is, we assume that the pulse occurs at $t=$ $(n+l) T(0<l<1)$ and $t=(n+1) T$. The detailed changes are shown as follows:

$$
\begin{gathered}
\dot{x}_{1}(t)=r x_{2}(t)-r e^{-\omega \tau} x_{2}(t-\tau)-\omega x_{1}(t), \\
\dot{x}_{2}(t)=r e^{-\omega \tau} x_{2}(t-\tau)-\beta x_{2}(t) y(t) \quad t \neq(n+l) T, \\
-d_{1} x_{2}(t)-d_{2} x_{2}^{2}(t), \\
\dot{y}(t)=k \beta x_{2}(t) y(t)-d_{3} y(t), \\
x_{1}\left(t^{+}\right)=\left(1-p_{0}\right) x_{1}(t), \quad t \neq(n+1) T, \\
x_{2}\left(t^{+}\right)=x_{2}(t), \\
y\left(t^{+}\right)=(1-p) y(t), \\
x_{1}\left(t^{+}\right)=x_{1}(t)+\mu, \\
x_{2}\left(t^{+}\right)=x_{2}(t), \\
b\left(t^{+}\right)=y(t)+\frac{b y(t)}{a+y(t)}, \quad t=(n+1) T, n \in Z^{+} . \\
\left(\omega_{1}(s), \omega_{2}(s), \omega_{3}(s)\right) \in C_{+}=C\left([-\tau, 0], R_{+}^{3}\right) \\
\omega_{i}(0)>0, \quad i=1,2,3 .
\end{gathered}
$$

If the mature prey $x_{2}(t)$ is absent, then system (22) reduces to

$$
\begin{aligned}
& \dot{x}_{1}(t)=-\omega x_{1}(t), \quad t \neq(n+l) T, \quad t \neq(n+1) T, \\
& \dot{y}(t)=-d_{3} y(t), \\
& x_{1}\left(t^{+}\right)=\left(1-p_{0}\right) x_{1}(t), \quad t=(n+l) T, \\
& y\left(t^{+}\right)=(1-p) y(t), \\
& x_{1}\left(t^{+}\right)=x_{1}(t)+\mu, \\
& y\left(t^{+}\right)=y(t)+\frac{b y(t)}{a+y(t)}, \quad t=(n+1) T, n \in Z^{+} .
\end{aligned}
$$

We can easily obtain the analytic solution of system (23) at the interval $[n T,(n+1) T)$ :

$$
\begin{aligned}
& x_{1}(t)=\left\{\begin{array}{l}
x_{1}\left(n T^{+}\right) e^{-\omega(t-n T)} \\
t \in(n T,(n+l) T], \\
x_{1}\left((n+l) T^{+}\right) e^{-\omega(t-(n+l) T)} \\
t \in((n+l) T,(n+1) T],
\end{array}\right. \\
& y(t)=\left\{\begin{array}{c}
y\left(n T^{+}\right) e^{-d_{3}(t-n T)} \\
t \in(n T,(n+l) T], \\
y\left((n+l) T^{+}\right) e^{-d_{3}(t-(n+l) T)} \\
t \in((n+l) T,(n+1) T] .
\end{array}\right.
\end{aligned}
$$

Lemma 5. (i) If $p>1-a e^{d_{3} T} /(a+b)$, the fixed point $y_{0}^{*}=0$ is globally asymptotically stable.

(ii) If $p<1-a e^{d_{3} T} /(a+b)$, the fixed point $y^{*}=((a+b)(1-$ $\left.p)-a e^{d_{3} T}\right) /(1-p)\left[1-(1-p) e^{-d_{3} T}\right]$ is globally asymptotically stable.
Proof. Consider system (24), which yields the following stroboscopic map:

$$
\begin{aligned}
y\left((n+1) T^{+}\right)= & (1-p) y\left(n T^{+}\right) e^{-d_{3} T} \\
& +\frac{b(1-p) y\left(n T^{+}\right) e^{-d_{3} T}}{a+(1-p) y\left(n T^{+}\right) e^{-d_{3} T}} .
\end{aligned}
$$

Let $F(y)=(1-p) y e^{-d_{3} T}+b(1-p) y e^{-d_{3} T} /\left(a+(1-p) y e^{-d_{3} T}\right)$. If $p>1-a e^{d_{3} T} /(a+b)$, there exists a unique trivial fixed point $y_{0}^{*}=0$. The trivial fixed point $y_{0}^{*}$ is locally stable if $p>1-a e^{d_{3} T} /(a+b)$, since

$$
\left|\frac{d F(y)}{d y}\right|_{y=0}=\frac{a(1-p) e^{-d_{3} T}+b(1-p) e^{-d_{3} T}}{a}<1,
$$

and unstable if $p<1-a e^{d_{3} T} /(a+b)$, since

$$
\left|\frac{d F(y)}{d y}\right|_{y=0}=\frac{a(1-p) e^{-d_{3} T}+b(1-p) e^{-d_{3} T}}{a}>1 .
$$

If $p<1-a e^{d_{3} T} /(a+b),(25)$ has a positive fixed point denoted by $y^{*}$, where $y^{*}=\left((a+b)(1-p)-a e^{d_{3} T}\right) /(1-p)[1-(1-$ p) $\left.e^{-d_{3} T}\right]$, when $p<1-a e^{d_{3} T} /(a+b)$. The positive fixed point $y^{*}$ is locally stable since $d F(y) /\left.(d y)\right|_{y=y^{*}}=\mid(1-p) e^{-d_{3} T}+$ $a\left[1-(1-p) e^{-d_{3} T}\right]^{2} / b(1-p) e^{-d_{3} T} \mid<1$, if $p<1-a e^{d_{3} T} /(a+b)$.

Further, we can show that $y^{*}$ is globally asymptotically stable, if the following statements are satisfied:

(i) if $y^{*}>y>0$, then $y^{*}>F(y)>y$;

(ii) if $y>y^{*}$, then $y>F(y)>y^{*}$.

By calculation, we get $d F / d y=(1-p) e^{-d_{3} T}+a b(1-$ p) $e^{-d_{3} T} /\left[a+(1-p) e^{-d_{3} T} y\right]^{2}>0$, which shows that $y^{*}>F(y)$, when $y^{*}>y>0$; we know

$$
\begin{aligned}
F(y)-y & =(1-p) y e^{-d_{3} T}+\frac{b(1-p) e^{-d_{3} T}}{a+(1-p) y e^{-d_{3} T}}-y \\
& >y\left((1-p) e^{-d_{3} T}+\frac{b(1-p) e^{-d_{3} T}}{a+(1-p) y^{*} e^{-d_{3} T}}-1\right) \\
& =0
\end{aligned}
$$

This yields $F(y)>y$. So we have $y^{*}>F(y)>y$ under the assumption $y^{*}>y>0$; otherwise $y>F(y)>y^{*}$ if $y>y^{*}$. Thus the statements (i) and (ii) are satisfied. This completes the proof.

Now we can deduce that the positive equilibrium $y^{*}$ of system (25) is globally asymptotically stable. So we have the 
corresponding positive periodic solution $\left(\tilde{x}_{1}(t), \tilde{y}(t)\right)$ in the following, which is globally stable:

$$
\begin{gathered}
\tilde{x}_{1}(t) \\
=\left\{\begin{array}{l}
\frac{\mu}{1-\left(1-p_{0}\right) e^{-\omega T}} e^{-\omega(t-n T)}, \quad t \in(n T,(n+l) T], \\
\frac{\mu\left(1-p_{0}\right)}{1-\left(1-p_{0}\right) e^{-\omega T}} e^{-\omega(t-n T)}, \quad t \in((n+l) T,(n+1) T],
\end{array}\right. \\
\tilde{y}(t)=\left\{\begin{array}{c}
\frac{(a+b)(1-p)-a e^{d_{3} T}}{(1-p)\left[1-(1-p) e^{-d_{3} T}\right]} e^{-d_{3}(t-n T)}, \\
t \in(n T,(n+l) T], \\
\frac{(a+b)(1-p)-a e^{d_{3} T}}{1-(1-p) e^{-d_{3} T}} e^{-d_{3}(t-n T)}, \\
t \in((n+l) T,(n+1) T] .
\end{array}\right.
\end{gathered}
$$

Theorem 6. Assume that

$$
R_{1}^{*}<1
$$

holds, where $R_{1}^{*}=\left(r e^{-\omega \tau}-d_{1}\right)\left[1-(1-p) e^{-d_{3} T}\right] / \beta[(1-p)(a+$ b) $\left.e^{-d_{3} T}-a\right]$. Then the mature prey-extinction periodic solution $\left(\widetilde{x}_{1}(t), 0, \widetilde{y}(t)\right)$ is globally asymptotically stable.

Proof. Following from the second equation of system (22), we notice that $\dot{y}(t) \geq-d_{3} y(t)$; thus consider the following impulsive differential system:

$$
\begin{gathered}
\dot{z}_{4}(t)=-d_{3} z_{4}(t), \quad t \neq(n+l) T, t \neq(n+1) T, \\
\Delta z_{4}(t)=-p z_{4}(t), \quad t=(n+l) T, \\
\Delta z_{4}(t)=\frac{b z_{4}(t)}{a+z_{4}(t)}, \quad t=(n+1) T, n \in Z^{+} .
\end{gathered}
$$

According to Lemma 5 and the comparison theorem on impulsive differential equations, we have $y(t) \geq z_{4}(t)$ and $z_{4}(t) \rightarrow \tilde{y}(t)$ as $t \rightarrow \infty$. Then there exists an integer $k_{2}>k_{1}$, $n>k_{2}$, such that

$$
y(t) \geq z_{4}(t) \geq \tilde{y}(t)-\varepsilon
$$

holds for all $t$ large enough.

This is

$$
\begin{aligned}
y(t) \geq \tilde{y}(t)-\varepsilon \\
\geq\left\{\begin{array}{c}
\frac{(a+b)(1-p)-a e^{d_{3} T}}{(1-p)\left[1-(1-p) e^{-d_{3} T}\right]} e^{-d_{3} l T}, \\
t \in(n T,(n+l) T], \\
\frac{(a+b)(1-p)-a e^{d_{3} T}}{1-(1-p) e^{-d_{3} T}} e^{-d_{3} T}, \\
t \in((n+l) T,(n+1) T] .
\end{array}\right.
\end{aligned}
$$

Since $\left(\left((a+b)(1-p)-a e^{d_{3} T}\right) /(1-p)\left[1-(1-p) e^{-d_{3} T}\right]\right) e^{-d_{3} l T}>$ $\left(\left((a+b)(1-p)-a e^{d_{3} T}\right) /\left(1-(1-p) e^{-d_{3} T}\right)\right) e^{-d_{3} T}$, we have

$$
\begin{array}{r}
y(t) \geq \frac{(a+b)(1-p)-a e^{d_{3} T}}{1-(1-p) e^{-d_{3} T}} e^{-d_{3} T}-\varepsilon \triangleq \psi, \\
t \in(n T,(n+1) T], n>k_{2} .
\end{array}
$$

For simplicity, we assume that (34) holds for all $t \geq 0$. From (22) we have

$$
\begin{aligned}
& \frac{d x_{2}(t)}{d t} \leq r e^{-\omega \tau} x_{2}(t-\tau) \\
&-\left(\beta(\tilde{y}(t)-\varepsilon)+d_{1}\right) x_{2}(t)-d_{2} x_{2}^{2}(t), \\
& t>n T+\tau, n>k_{2} .
\end{aligned}
$$

Consider the following comparison differential system:

$$
\begin{aligned}
& \frac{d z_{5}(t)}{d t}= r e^{-\omega \tau} z_{5}(t-\tau) \\
&-\left(\beta(\tilde{y}(t)-\varepsilon)+d_{1}\right) z_{5}(t)-d_{2} z_{5}^{2}(t), \\
& t>n T+\tau, n>k_{2} .
\end{aligned}
$$

From (23) and Lemma 1, we have $\lim _{t \rightarrow \infty} z_{5}(t)=0$. By the comparison differential theorem, we have $\lim _{t \rightarrow \infty} x_{2}(t)<$ $\lim _{t \rightarrow \infty} z_{5}(t)=0$. Noticing the positivity of $x_{2}(t)$, we know that $\lim _{t \rightarrow \infty} x_{2}(t)=0$. Without loss of generality, we assume that there exists an integer $k_{3}, k_{3}>k_{2}$, for any $\varepsilon_{1}$ (sufficiently small), such that

$$
x_{2}(t) \leq \varepsilon_{1} .
$$

In view of (22) and (37), it is easy to obtain that

$$
-r e^{-\omega \tau} \varepsilon_{1}-\omega x_{1}(t) \leq \dot{x}_{1}(t) \leq r \varepsilon_{1}-\omega x_{1}(t),
$$

we get $z_{6}(t) \leq x_{1}(t) \leq z_{7}(t)$, and $z_{6}(t) \rightarrow \tilde{x}_{1}(t), z_{7}(t) \rightarrow$ $\tilde{x}_{1}(t)$ as $t \rightarrow \infty$, where

$$
\begin{gathered}
\dot{z}_{6}(t)=-r e^{-\omega \tau} \varepsilon_{1}-\omega z_{6}(t), \\
t \neq(n+l) T, t \neq(n+1) T, \\
z_{6}\left(t^{+}\right)=\left(1-p_{0}\right) z_{6}(t), \quad t=(n+l) T, \\
z_{6}\left(t^{+}\right)=z_{6}(t)+\frac{b z_{6}(t)}{a+z_{6}(t)}, \quad t=(n+1) T, n \in Z^{+}, \\
\dot{z}_{7}(t)=r \varepsilon_{1}-\omega z_{7}(t), \quad t \neq(n+l) T, t \neq(n+1) T, \\
z_{7}\left(t^{+}\right)=\left(1-p_{0}\right) z_{7}(t), \quad t=(n+l) T, \\
z_{7}\left(t^{+}\right)=z_{7}(t)+\frac{b z_{7}(t)}{a+z_{7}(t)}, \quad t=(n+1) T, n \in Z^{+} .
\end{gathered}
$$

From the comparison differential theorem, for any $\varepsilon_{2}$, there exists an integer $k_{4}, n>k_{4}$; let $\varepsilon_{1} \rightarrow 0$; then $\widetilde{z}_{6}(t)-\varepsilon_{2}<$ 
$x_{1}(t)<\widetilde{z}_{7}(t)+\varepsilon_{2}$, for $t$ large enough, which means $x_{1}(t) \rightarrow$ $\tilde{x}_{1}(t)$ as $t \rightarrow \infty$, where

$$
\begin{aligned}
& \tilde{z}_{6}(t) \\
& =\left\{\begin{array}{c}
\frac{-r e^{-\omega \tau} \varepsilon_{1}}{\omega} \\
+\left(\frac{\mu}{1-\left(1-p_{0}\right) e^{-\omega T}}-\frac{-r e^{-\omega \tau} \varepsilon_{1}}{\omega}\right) e^{-\omega(t-n T)}, \\
\frac{-r e^{-\omega \tau} \varepsilon_{1}}{\omega} \\
+\left(\frac{\mu(n T,(n+l) T],}{\left.1-\left(1-p_{0}\right) e^{-\omega l T}\right) e^{-\omega T}}-\frac{-r e^{-\omega \tau} \varepsilon_{1}}{\omega}\right) e^{-\omega(t-(n+l) T)}, \\
t \in((n+l) T,(n+1) T],
\end{array}\right. \\
& \widetilde{z}_{7}(t)=\left\{\begin{array}{l}
\frac{r \varepsilon_{1}}{\omega}+\left(\frac{\mu}{1-\left(1-p_{0}\right) e^{-\omega T}}-\frac{r \varepsilon_{1}}{\omega}\right) e^{-\omega(t-n T)}, \\
t \in(n T,(n+l) T] \\
\frac{r \varepsilon_{1}}{\omega}+\left(\frac{\mu\left(1-p_{0}\right) e^{-\omega l T}}{1-\left(1-p_{0}\right) e^{-\omega T}}-\frac{r \varepsilon_{1}}{\omega}\right) e^{-\omega(t-(n+l) T)} \\
t \in((n+l) T,(n+1) T]
\end{array}\right.
\end{aligned}
$$

Next, we will prove that $y(t) \rightarrow \tilde{y}(t)$ as $t \rightarrow \infty$. From (22) and (37), we get

$$
-d_{3} y(t) \leq \dot{y}(t) \leq-\left(d_{3}-\lambda \beta \varepsilon_{1}\right) y(t) .
$$

For the left hand inequality, it follows from impulsive differential equation (31) that $y(t) \geq z_{4}(t)$ and $z_{4}(t) \rightarrow \tilde{y}_{1}(t)$ as $t \rightarrow \infty$. For the right hand inequality, we consider the following impulsive differential equation:

$$
\begin{gathered}
\dot{z}_{8}(t)=-\left(d_{3}-\lambda \beta \varepsilon_{1}\right) z_{8}(t), \\
t \neq(n+l) T, \quad t \neq(n+1) T, \\
\Delta z_{8}(t)=-p z_{8}(t), \quad t=(n+l) T, \\
\Delta z_{8}(t)=\frac{b z_{8}(t)}{a+z_{8}(t)}, \quad t=(n+1) T, n \in Z^{+}, \\
\widetilde{z}_{8}(t)=\left\{\begin{array}{c}
(a+b)(1-p)-a e^{\left(d_{3}-\lambda \beta \varepsilon_{1}\right) T} \\
\frac{(1-p)\left[1-(1-p) e^{-\left(d_{3}-\lambda \beta \varepsilon_{1}\right) T}\right.}{(a+b)(1-p)-a e^{\left(d_{3}-\lambda \beta \varepsilon_{1}\right) T}} e^{-\left(d_{3}-\lambda \beta \varepsilon_{1}\right)(t-n T)}, \\
\frac{(n T,(n+l) T],}{1-(1-p) e^{-\left(d_{3}-\lambda \beta \varepsilon_{1}\right) T}} e^{\left.-\lambda \beta \varepsilon_{1}\right)(t-n T)}, \\
t \in((n+l) T,(n+1) T] .
\end{array}\right.
\end{gathered}
$$

Therefore, for any $\varepsilon_{3}$, there exists an integer $k_{5}, n>k_{5}$ such that

$$
\tilde{z}_{4}(t)-\varepsilon_{3}<y(t)<\widetilde{z}_{8}(t)+\varepsilon_{3} .
$$

Let $\varepsilon_{1} \rightarrow 0$; then we get

$$
\tilde{y}(t)-\varepsilon_{3}<y(t)<\tilde{y}(t)+\varepsilon_{3},
$$

for $t$ large enough, which means $y(t) \rightarrow \tilde{y}(t)$ as $t \rightarrow \infty$. The proof is completed.
Corollary 7. (i) If $p<1-\left[\left(r e^{-\omega \tau}-d_{1}\right)+a \beta\right] e^{d_{3} T} /\left(\left(r e^{-\omega \tau}-\right.\right.$ $\left.\left.d_{1}\right)+(a+b) \beta\right)$ holds, then the mature prey-extinction periodic solution $\left(\tilde{x}_{1}(t), 0, \tilde{y}(t)\right)$ is globally asymptotically stable.

(ii) If $T<\left(1 / d_{3}\right) \ln \left(\left[\left(r e^{-\omega \tau}-d_{1}\right)+(a+b) \beta\right](1-p) /\left[\left(r e^{-\omega \tau}-\right.\right.\right.$ $\left.\left.\left.d_{1}\right)+a \beta\right] e^{d_{3} T}\right)$ holds, then the mature prey-extinction periodic solution $\left(\tilde{x}_{1}(t), 0, \tilde{y}(t)\right)$ is globally asymptotically stable.

The biological significance of Corollary 7 is the same as Corollary 4, so we omit it.

In order to investigate the permanence of system (22), we should give the following Definition and Lemma.

Lemma 8. There exists a constant $M>0$ such that $x_{1}(t) \leq M / k, x_{2}(t) \leq M / k, y(t) \leq M$ for each solution $\left(x_{1}(t), x_{2}(t), y(t)\right)$ of system (22) with all $t$ large enough.

Proof. Define $V(t)=k x_{1}(t)+k x_{2}(t)+y(t)$, note that $\omega>d_{3}$, then $t \neq(n+l) T, t \neq(n+1) T$ and we have

$$
\begin{aligned}
D^{+} V( & t)+d_{3} V(t) \\
& =-k\left(\omega-d_{3}\right) x_{1}(t)+k\left(r+d_{3}-d_{1}\right) x_{2}(t)-k d_{2} x_{2}^{2}(t) \\
& \leq-k d_{2}\left(x_{2}^{2}(t)-\frac{r+d_{3}-d_{1}}{2 d_{2}}\right)^{2}+\frac{k\left(r+d_{3}-d_{1}\right)^{2}}{4 d_{2}} \\
& \leq \frac{k\left(r+d_{3}-d_{1}\right)^{2}}{4 d_{2}} \triangleq M_{1} .
\end{aligned}
$$

When $t=(n+l) T$,

$$
\begin{aligned}
V( & \left.(n+l) T^{+}\right) \\
= & k x_{1}\left((n+l) T^{+}\right)+k x_{2}\left((n+l) T^{+}\right)+y\left((n+l) T^{+}\right) \\
= & k\left(\left(1-p_{0}\right)\right) x_{1}((n+l) T) \\
& +k x_{2}((n+l) T)+(1-p) y((n+l) T) \\
\leq & V((n+l) T) .
\end{aligned}
$$

When $t=(n+1) T$,

$$
\begin{aligned}
& V\left((n+1) T^{+}\right) \\
& \quad=k x_{1}\left((n+1) T^{+}\right)+k x_{2}\left((n+1) T^{+}\right)+y\left((n+1) T^{+}\right) \\
& \quad=V((n+1) T)+k \mu+\frac{b y((n+1) T)}{a+y((n+1) T)} \\
& \quad \leq V((n+1) T)+k \mu+b
\end{aligned}
$$

Let us denote $M_{2}=k \mu+b$. 
It follows from the comparison theorem of impulsive differential equations (see lemma 2.2 [25], page 23) that

$$
\begin{array}{r}
V(t) \leq V\left(0^{+}\right) e^{-d_{3} t}+M_{1} \frac{1-e^{-d_{3} t}}{d_{3}}+M_{2} \frac{e^{-d_{3}(t-T)}}{1-e^{d_{3} T}} \\
+M_{2} \frac{e^{d_{3} T}}{e^{d_{3} T}-1} \longrightarrow \frac{M_{1}}{d_{3}}+M_{2} \frac{e^{d_{3} T}}{e^{d_{3} T}-1}, \\
\text { as } t \longrightarrow \infty .
\end{array}
$$

So $V(t)$ is ultimately bounded. Hence, by the definition of $V(t)$, there exists a constant $M>0$, such that $x_{1}(t) \leq$ $M / k, \quad x_{2}(t) \leq M / k, y(t) \leq M$ for all $t$ large enough. This completes the proof.

Remark 9. According to Lemma 8, it is clear that $\limsup _{t \rightarrow \infty} V(t) \rightarrow M_{1} / d_{3}+M_{2}\left(e^{d_{3} T} /\left(e^{d_{3} T}-1\right)\right)$. For convenience, we note that $M=\left(k\left(r+d_{3}-d_{1}\right)^{2} / 4 d_{2} d_{3}\right)+$ $(k \mu+b)\left(e^{d_{3} T} /\left(e^{d_{3} T}-1\right)\right)$.

Definition 10. The system (22) is said to be permanent if there are constants $m, M>0$ (independent of all initial values) and a finite time $T_{0}$, such that for all solutions $\left(x_{1}(t), x_{2}(t), y(t)\right)$ with all initial values $x_{1}\left(0^{+}\right)>0, x_{2}\left(0^{+}\right)>0, y\left(0^{+}\right)>0, m \leq$ $x_{1}(t) \leq M / k, m \leq x_{2}(t) \leq M / k$, and $m \leq y(t) \leq M$ holds for all $t \geq T_{0}$.

In biological terms, the permanence of (22) implies that prey (both immature and mature) and predators will coexist, none of them facing extinction or growing indefinitely. Denote

$$
\begin{aligned}
R^{*} & =\frac{(a+b)(1-p)-a e^{\left(d_{3}-k \beta \theta\right) T}}{(1-p)\left[1-(1-p) e^{-\left(d_{3}-k \beta \theta\right) T}\right]}, \\
\theta & =\frac{(a+b)(1-p)}{a e^{d_{3} T}}\left(1-\frac{1}{R_{2}^{*}}\right)+\frac{1}{R_{2}^{*}} .
\end{aligned}
$$

Theorem 11. If

$$
R_{2}^{*}>1
$$

holds, where $R_{2}^{*}=\left(\left(r e^{-\omega \tau}-d_{1}-d_{2}(M / k)\right) / \beta R^{*}\right)$, system (22) is permanent.

Proof. It is seen that the second equation of system (22) can be rewritten as

$$
\begin{aligned}
\frac{d x_{2}(t)}{d t}= & \left(r e^{-\omega \tau}-\beta y(t)-d_{1}-d_{2} x_{2}(t)\right) x_{2}(t) \\
& -r e^{-\omega \tau} \frac{d}{d t} \int_{t-\tau}^{t} x_{2}(u) d u .
\end{aligned}
$$

Let us consider any positive solution $\left(x_{1}(t), x_{2}(t), y(t)\right)$ of system (22). By (51), $V(t)$ is defined as

$$
V(t)=x_{2}(t)+r e^{-\omega \tau} \frac{d}{d t} \int_{t-\tau}^{t} x_{2}(u) d u
$$

Taking the first derivative of $V(t)$ with respect to $t$, we have

$$
\frac{d V(t)}{d t}=\left(r e^{-\omega \tau}-\beta y(t)-d_{1}-d_{2} x_{2}(t)\right) x_{2}(t) .
$$

As seen in Lemma 8, (53) can be written as

$$
\frac{d V(t)}{d t}>\left(r e^{-\omega \tau}-\beta y(t)-d_{1}-d_{2} \frac{M}{k}\right) x_{2}(t) .
$$

From the definition of $R_{2}^{*}$, we have

$$
e^{k \beta T \theta}=\frac{(a+b)(1-p)}{a e^{d_{3} T}}\left(1-\frac{1}{R_{2}^{*}}\right)+\frac{1}{R_{2}^{*}}>1,
$$

and then $\theta>0$; it is easy to know that there exists a sufficiently small $\varepsilon$ such that

$$
r e^{-\omega \tau}>\beta\left(R^{*}+\varepsilon\right)+d_{1}+d_{2} \frac{M}{k} .
$$

We claim that, for any $t_{0}>0$, it is impossible that $x_{2}(t)<\theta$ for all $t>t_{0}$. Otherwise, there is a $t_{0}>0$ such that $\theta$ for all $t>t_{0}$. It follows from the third equation of (22) that

$$
\dot{y}(t)<\left(k \beta \theta-d_{3}\right) y(t),
$$

for all $t>t_{0}$. Consider the following comparison impulsive system of (57):

$$
\begin{gathered}
\dot{z}_{9}(t)=-\left(d_{3}-k \beta \theta\right) z_{9}(t), \\
t \neq(n+l) T, \quad t \neq(n+1) T, \\
\Delta z_{9}(t)=-p z_{9}(t), \quad t=(n+l) T, \\
\Delta z_{9}(t)=\frac{b z_{9}(t)}{a+z_{9}(t)}, \quad t=(n+1) T, n \in Z^{+},
\end{gathered}
$$

and we have

$$
\widetilde{z}_{9}(t)=\left\{\begin{array}{c}
\frac{(a+b)(1-p)-a e^{\left(d_{3}-k \beta \theta\right) T}}{(1-p)\left[1-(1-p) e^{-\left(d_{3}-k \beta \theta\right) T}\right]} e^{-\left(d_{3}-k \beta \theta\right)(t-n T)}, \\
t \in(n T,(n+l) T], \\
\frac{(a+b)(1-p)-a e^{\left(d_{3}-k \beta \theta\right) T}}{\left[1-(1-p) e^{-\left(d_{3}-k \beta \theta\right)}\right]} e^{-\left(d_{3}-k \beta \theta\right)(t-n T)}, \\
t \in((n+l) T,(n+1) T] .
\end{array}\right.
$$

In view of the comparison differential theorem for equation, there exists a $T_{1}>0$, such that

$$
\begin{aligned}
y(t) & <z_{9}(t)<\widetilde{z}_{9}(t)+\varepsilon_{1} \\
& \leq\left\{\begin{array}{c}
\frac{(a+b)(1-p)-a e^{\left(d_{3}-k \beta \theta\right) T}}{(1-p)\left[1-(1-p) e^{-\left(d_{3}-k \beta \theta\right) T}\right]}+\varepsilon_{1}, \\
t \in(n T,(n+l) T], \\
e^{-\left(d_{3}-k \beta \theta\right) l T} \frac{(a+b)(1-p)-a e^{\left(d_{3}-k \beta \theta\right) T}}{\left[1-(1-p) e^{-\left(d_{3}-k \beta \theta\right)}\right]}+\varepsilon_{1}, \\
t \in((n+l) T,(n+1) T],
\end{array}\right.
\end{aligned}
$$


for $t>T_{1}$, which implies that $y(t) \leq \widetilde{z}_{9}(t)+\varepsilon_{1}$, where

$$
\widetilde{z}_{9}(t) \leq \frac{(a+b)(1-p)-a e^{\left(d_{3}-k \beta \theta\right) T}}{(1-p)\left[1-(1-p) e^{-\left(d_{3}-k \beta \theta\right) T}\right]}=R^{*} .
$$

Thus

$$
y(t) \leq R^{*}+\varepsilon_{1} \triangleq \eta, \quad t>T_{1} .
$$

From (54), we get

$$
r e^{-\omega \tau}>\beta \eta+d_{1}+d_{2} \frac{M}{k}
$$

By (54) and (63), we have

$$
\dot{V}(t)>\left(r e^{-\omega \tau}-\beta \eta-d_{1}-d_{2} \frac{M}{k}\right) x_{2}(t), \quad t \geq T_{1} .
$$

Let

$$
x_{2}^{m}=\min _{t \in\left[T_{1}, T_{1}+\tau\right]} x_{2}(t)
$$

In the following we show that $x_{2}(t) \geq x_{2}^{m}$ for all $t \geq T_{1}$. Otherwise, there is a $T_{2}>0$ such that $x_{2}(t) \geq x_{2}^{m}$ for $T_{1} \leq t \leq$ $T_{1}+\tau+T_{2}, x_{2}\left(T_{1}+\tau+T_{2}\right)=x_{2}^{m}$ and $\dot{x}_{2}\left(T_{1}+\tau+T_{2}\right)<0$, which implies that,

$$
\begin{aligned}
\dot{x}_{2} & \left(T_{1}+T_{2}+\tau\right) \\
= & r e^{-\omega \tau} x_{2}\left(T_{1}+T_{2}+\tau\right) \\
& -\beta x_{2}\left(T_{1}+T_{2}+\tau\right) y\left(T_{1}+T_{2}+\tau\right) \\
& -d_{1} x_{2}\left(T_{1}+T_{2}+\tau\right)-d_{2} x_{2}^{2}\left(T_{1}+T_{2}+\tau\right) \\
\geq & \left(r e^{-\omega \tau}-\beta \eta-d_{1}-d_{2} M\right) x_{2}^{m}>0,
\end{aligned}
$$

it is a contradiction. Thus, $x_{2} \geq x_{2}^{m}$ for all $t \geq T_{1}$. Hence, for $t \geq T_{1}$, we get

$$
\dot{V}(t)>x_{2}^{m}\left(r_{1} e^{-\omega \tau}-\beta \eta-d_{1}-d_{2} M\right)>0,
$$

which means $V(t) \rightarrow \infty$ as $t \rightarrow \infty$. This is contrary to the boundedness of $V(t)$. The claim is proved.

By the claim, consider the following two possibilities.

Case 1. $x_{2}(t) \geq x_{2}^{*}$ for all $t$ large enough.

Case 2. $x_{2}$ oscillates about $x_{2}^{*}$ for $t$ large enough. Set

$$
q=\min \left\{\frac{x_{2}^{*}}{2}, x_{2}^{*} e^{-\left(\beta M+d_{1}+d_{2} x_{2}^{*}\right) \tau}\right\}
$$

We will show that $x_{2}(t) \geq q$ for all $t$ large enough. The conclusion is evident in Case 1. For Case 2, suppose that there exists the positive constant $\bar{t}, \xi$ satisfying $x_{2}(\bar{t})=x_{2}(\bar{t}+\xi)=$ $x_{2}^{*}$ and $x_{2}(t)<x_{2}^{*}$ for all $\bar{t}<t<\bar{t}+\xi$, where $\bar{t}$ is enough large such that

$$
x_{2}(t)>q \text { for } \bar{t}<t<\bar{t}+\xi
$$

since $x_{2}(t)$ is continuous and bounded and is not affected by impulses. Thus $x_{2}(t)$ is uniformly continuous. Consequently, there exists a constant $T_{3}\left(0<T_{3}<\tau\right.$ and $T_{3}$ is independent of the choice of $\bar{t})$ such that $x_{2}(t)>\left(x_{2}^{*} / 2\right)$ for $\bar{t} \leq t \leq \bar{t}+T_{3}$. If $\xi \leq T_{3}$, our aim is obtained. Then we consider the case $T_{3}<\xi<\tau$. From the second equation of (22) and the above assumption, we have that $\dot{x}_{2}(t) \geq-\left(\beta M+d_{1}+d_{2} x_{2}^{*}\right) x_{2}(t)$ for $\bar{t}<t \leq \bar{t}+\xi \leq \bar{t}+\tau$. Hence one obtains $x_{2}(t) \geq$ $x_{2}^{*} e^{-\left(\beta M+d_{1}+d_{2} x_{2}^{*}\right) \tau}$. It is clear that $x_{2}(t) \geq q$ for $\bar{t}<t \leq \bar{t}+\xi$. If $\xi \geq \tau$, then we have $x_{2}(t) \geq q$ for $\bar{t}<t \leq \bar{t}+\tau$. The same arguments can be continued; we can obtain $x_{2}(t) \geq q$ for $\bar{t}+\tau<t \leq \bar{t}+\xi$. Since the kind of interval $[\bar{t}, \bar{t}+\xi]$ is chosen in arbitrary way, we have $x_{2}(t) \geq q$ for $t$ large enough. In view of the above discussion, the selection of $q$ is independent of the positive solution of (22) which satisfies $x_{2}(t) \geq q$ for all $t$ large enough.

Next, from the first, the fourth, and the seventh equation of system (22), we have

$$
\begin{gathered}
\dot{x}_{1}(t) \geq-\omega x_{1}(t), \quad t \neq(n+l) T, \quad t \neq(n+1) T, \\
\Delta x_{1}(t)=-p_{0} x_{1}(t), \quad t=(n+l) T, \\
\Delta x_{1}(t)=\mu, \quad t=(n+1) T, n \in Z^{+} .
\end{gathered}
$$

Considering the comparison system,

$$
\begin{gathered}
\dot{z}_{10}(t)=-\omega z_{10}(t), \quad t \neq(n+l) T, \quad t \neq(n+1) T, \\
\Delta z_{10}(t)=-p_{0} z_{10}(t), \quad t=(n+l) T, \\
\Delta z_{10}(t)=\mu, \quad t=(n+1) T, n \in Z^{+} .
\end{gathered}
$$

Therefore for any $\varepsilon_{4}>0$ small enough such that $x_{1}(t) \geq$ $\widetilde{z}_{10}(t)-\varepsilon_{4}$ for $t$ sufficiently large,

$$
\widetilde{z}_{10}(t)=\left\{\begin{array}{c}
\frac{\mu}{1-\left(1-p_{0}\right) e^{-\omega T}} e^{-\omega(t-n T)}, \\
t \in(n T,(n+l) T], \\
\frac{\mu\left(1-p_{0}\right)}{1-\left(1-p_{0}\right) e^{-\omega T}} e^{-\omega(t-n T)}, \\
t \in((n+l) T,(n+1) T]
\end{array}\right.
$$

In view of the comparison theorem of impulsive differential equation and the Theorem 6 , we get

$$
x_{1}(t) \geq \frac{\mu\left(1-p_{0}\right)}{1-\left(1-p_{0}\right) e^{-\omega T}} e^{-\omega T}-\varepsilon_{2} \triangleq m_{1} .
$$

From (34) and (68), set $\psi \triangleq m_{3}$ and $q \triangleq m_{2}$.

Define $m=\min \left\{m_{1}, m_{2}, m_{3}\right\}$, and thus we have $x_{1}(t) \geq$ $m, x_{2}(t) \geq m, y(t) \geq m$. By Lemma 8 and the above discussion, the system is permanent. The proof is completed.

\section{Numerical Analysis}

In the previous sections, we introduced the analytical tools and used them for a qualitative analysis of the system obtaining some results about the dynamics of the system. In this section, we perform a numerical analysis of the model 


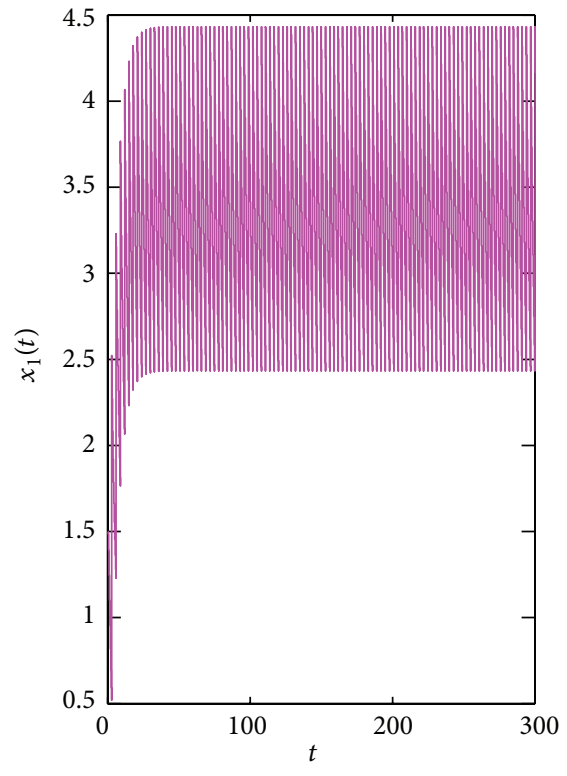

(a)

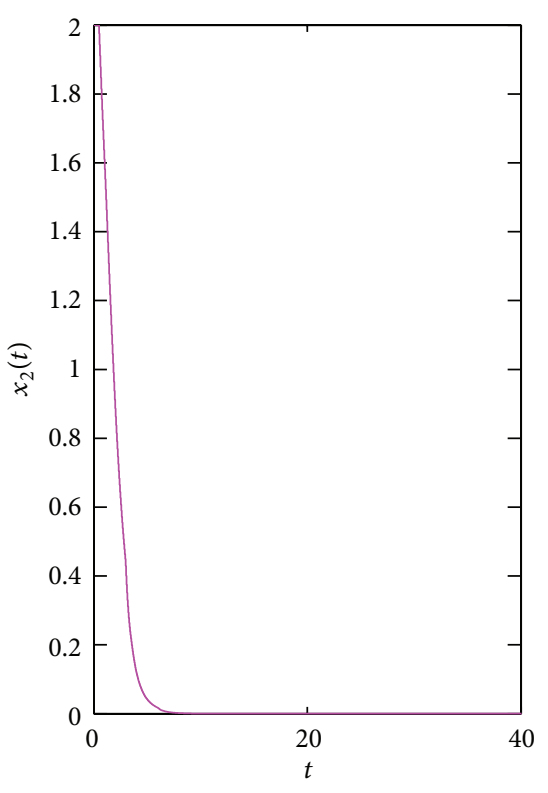

(b)

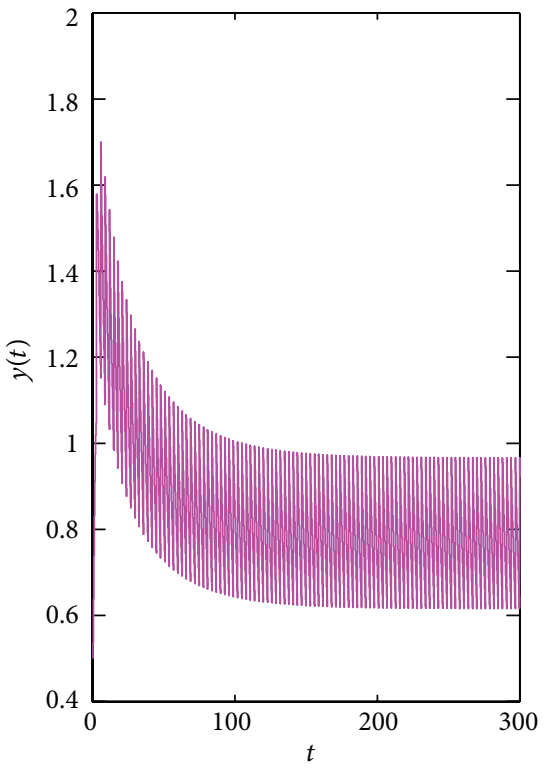

(c)

FIGURE 1: Dynamical behavior of system (1); the parameter values are as follows: $r=1.2, \tau=0.5, \omega=0.2, \beta=2, \mu=2, k=0.2, d_{1}=0.1, d_{2}=$ $0.2, d_{3}=0.15, p_{0}=0.1, p=0.5, a=5, b=6$, and $T=3$.

based on the previous results. What we are interested in is how the key factors affect the thresholds $R_{1}$ and $R_{1}^{*}$. Let $R_{1}(p, T, \tau)=\left(\left(r e^{-\omega \tau}-d_{1}\right)\left[1-(1-p) e^{-d_{3} T}\right] / \beta\left[a(1-p) e^{-d_{3} T}+\right.\right.$ $\left.\left.b e^{-d_{3} T}-a\right]\right)$. Taking the first derivatives $R_{1}(p, T, \tau)$ with respect to $p, T$ and $\tau$, respectively, one obtains

$$
\begin{gathered}
\frac{\partial R_{1}}{\partial p}=\frac{\left(r e^{-\omega \tau}-d_{1}\right)}{\beta} \frac{b e^{-2 d_{3} T}}{\left[a(1-p) e^{-d_{3} T}+b e^{-d_{3} T}-a\right]^{2}}>0, \\
\frac{\partial R_{1}}{\partial T}=\frac{\left(r e^{-\omega \tau}-d_{1}\right)}{\beta} \frac{d_{3} b e^{-d_{3} T}}{\left[a(1-p) e^{-d_{3} T}+b e^{-d_{3} T}-a\right]^{2}}>0, \\
\frac{\partial R_{1}}{\partial \tau}=-\omega \frac{r e^{-\omega \tau}\left[1-(1-p) e^{-d_{3} T}\right]}{\beta\left[a(1-p) e^{-d_{3} T}+b e^{-d_{3} T}-a\right]}<0 .
\end{gathered}
$$

Those inequalities demonstrate that threshold value $R_{1}$ is a monotonic increasing function with respect to $p$ and $T$ and is a monotonic decreasing function with $\tau$. These results indicate that the smaller killing (or poisoning) rate $p$ or the shorter pulse period $T$, the smaller the threshold value $R_{1}$ which follows, so, the result shows that it is more effective for pest control. Similarly, when pests have very short maturity $\tau$, the bigger the threshold value $R_{1}$ which follows, and hence make it more difficult for pest control. Therefore, we must carefully select the impulsive catching (or poisoning) rate or the time of pesticide applications or reduce the dosages of the pesticide.

So far, we have considered the global attractive mature prey-eradication solution of systems (1) and (22). We first focus on the system (1); when threshold value $R_{1}<1$, the mature prey is eradicated totally and the immature prey and the predator population will tend to a stable level; see Figure 1, where $r=1.2, \tau=0.5, \omega=0.2, \beta=2, \mu=2, k=0.2, d_{1}=$ $0.1, d_{2}=0.2, d_{3}=0.15, p_{0}=0.1, p=0.5, a=5, b=6, T=3$, and $R_{1} \approx 0.7997<1$, while if we chose the parameters set as those in Figure 2, then the all populations oscillate periodically, which indicates that the system (1) is permanent. Furthermore, we can carry out two-parameter bifurcation analysis for the threshold value $R_{1}$, as those shown in Figures 3(a)-3(d). In each subplot, we let two key parameters vary simultaneously and see how those parameters affect the threshold value $R_{1}$. All simulation results shown in Figure 3 indicate that the $R_{1}$ appears to be quite sensitive to small changes in parameters, $p, T, \tau$, and $b$. However, we note that the dynamical behaviour of system (1) is dominated by several types of periodic solutions and their coexistence, even with very high intrinsic growth rates. In Figure 4, we studied the influence of killing rate $p$ on the complexity of system (1); numerical results show that when killing rate $p$ varies from 0.45 to 0.95 , the dynamical behaviour of system (1) is very complex. A typical chaos oscillation is captured when $p=0.7$ (see Figure 5). Figure 6 shows the bifurcation diagrams of system (1); the system analyzed here could take on many forms of complexity, including period doubling bifurcation, symmetry-breaking pitchfork bifurcation, and chaotic solutions. Meanwhile, system (1) also has a certain type of coexistence if the intrinsic growth rate of the pest population is large enough.

For system (22), according to Theorem 6, we know that, if $R_{1}^{*}<1$, the mature prey-extinction periodic solution is 


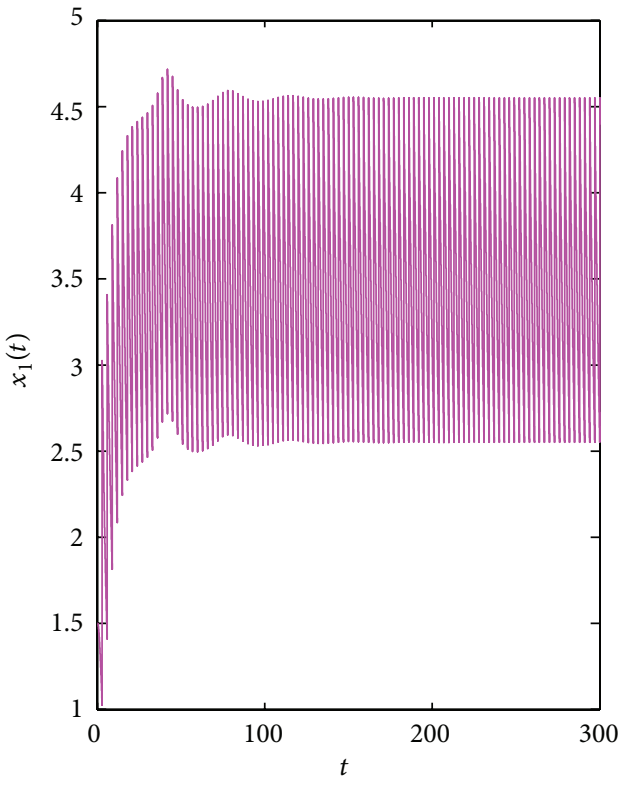

(a)

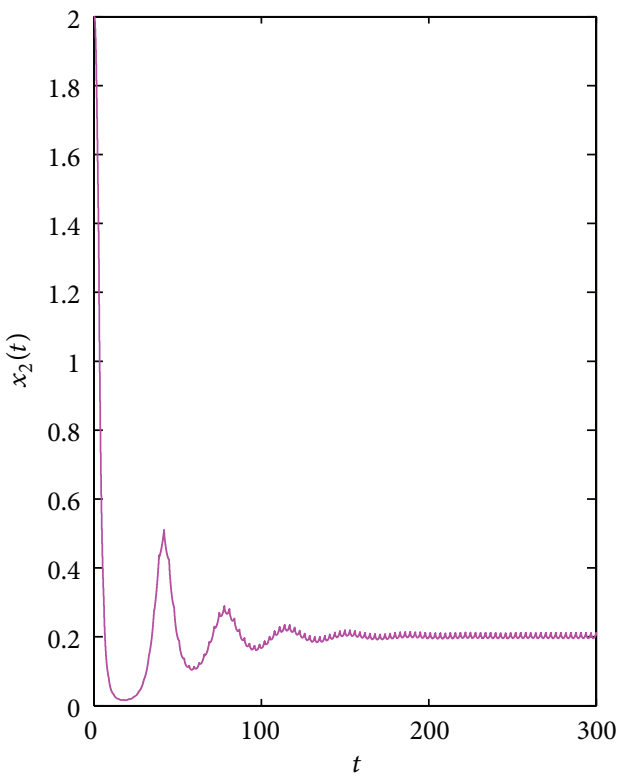

(b)

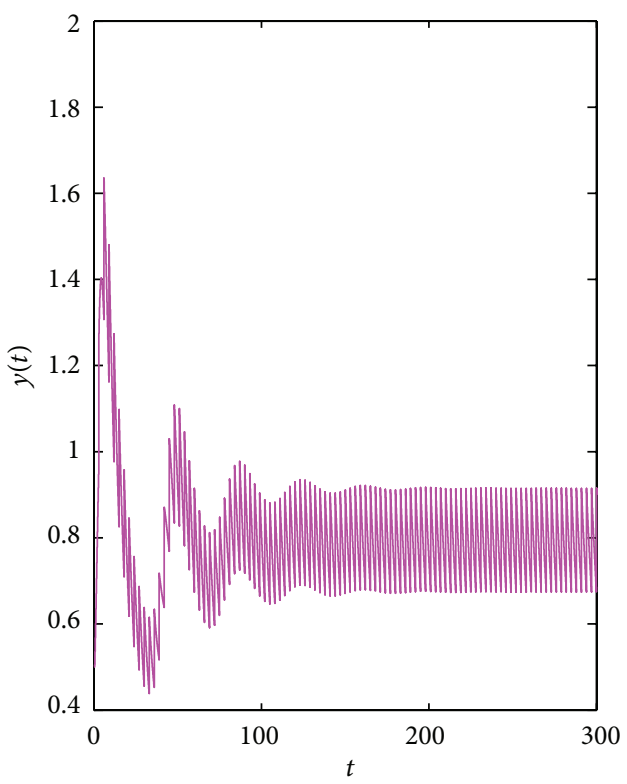

(c)

Figure 2: Dynamical behavior of system (1); the parameter values are as follows: $r=1.2, \tau=0.5, \omega=0.2, \beta=1.2, k=0.2, d_{1}=0.1, d_{2}=$ $0.2, d_{3}=0.15, p_{0}=0.1, p=0.7, a=5, b=6, \mu=2$, and $T=3$.

globally attractive. This can be seen clearly from Figure 7, where $r=5, \tau=0.5, \omega=0.2, \beta=0.6, k=0.2, d_{1}=$ $0.15, d_{2}=0.2, d_{3}=0.1, p_{0}=0.2, p=0.5, a=3, b=6, T=$ $2, l=0.7$, and $\mu=2$; then $R_{1}^{*} \approx 0.8733<1$, while if we chose the parameters set as those in Figure 8, it is clear that the system (22) is permanent. By using the same methods as those for system (1), we can investigate the effects of killing rate $p$, pulse periodic $T$, birth rate $r$ of pest populations, and the biggest birth rate $b$ of predator on the threshold value $R_{1}^{*}$ and discuss their biological implications, so we omitted them here.

We are more interested in how different patterns of insecticide applications affect the two threshold values $\left(R_{1}, R_{1}^{*}\right)$ and which strategy is more conducive to pest control. To do this, we investigate the superiority of the two strategies. Assume that the $R_{1}$ and $R_{1}^{*}$ are with the same parameters; Figure 9 provides the details of how different control strategies affect the threshold values $R_{1}$ and $R_{1}^{*}$. If $p=0$, that 


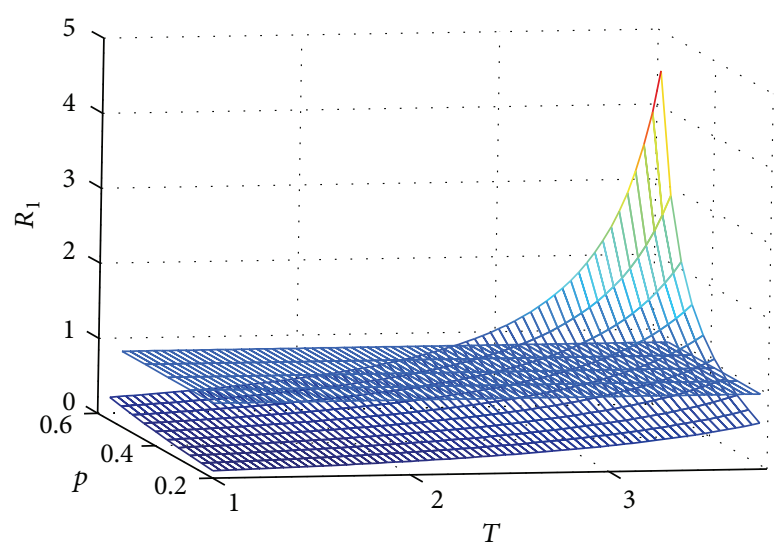

(a)

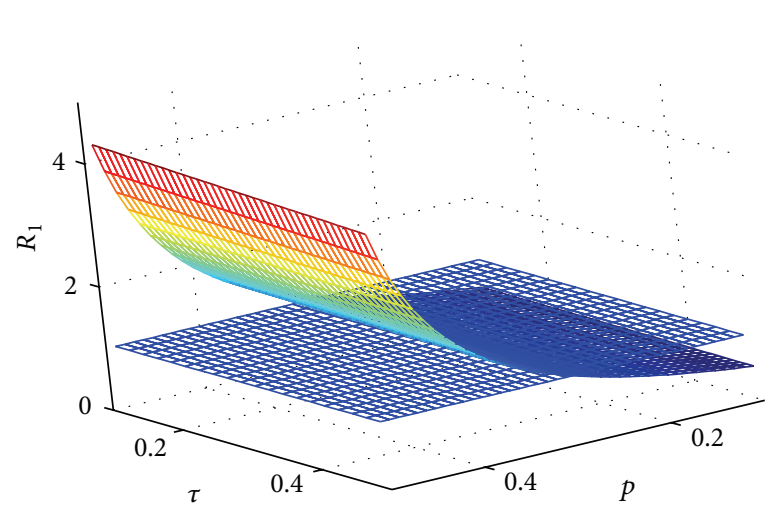

(c)

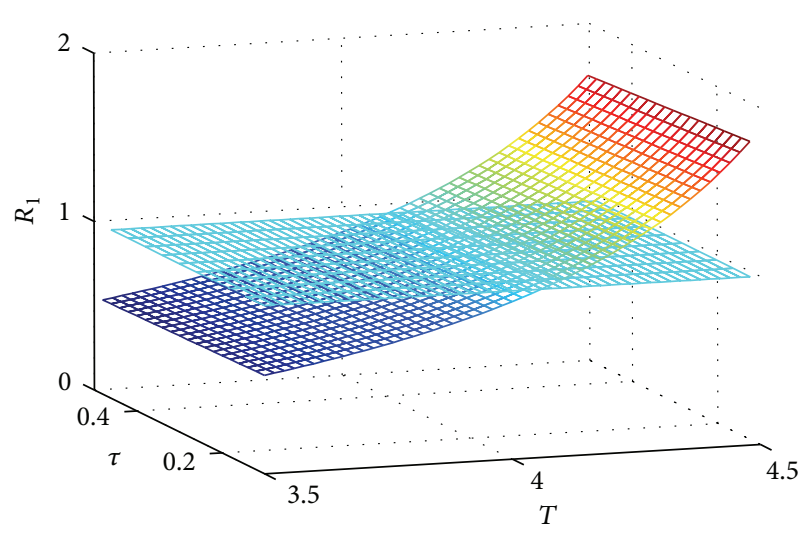

(b)

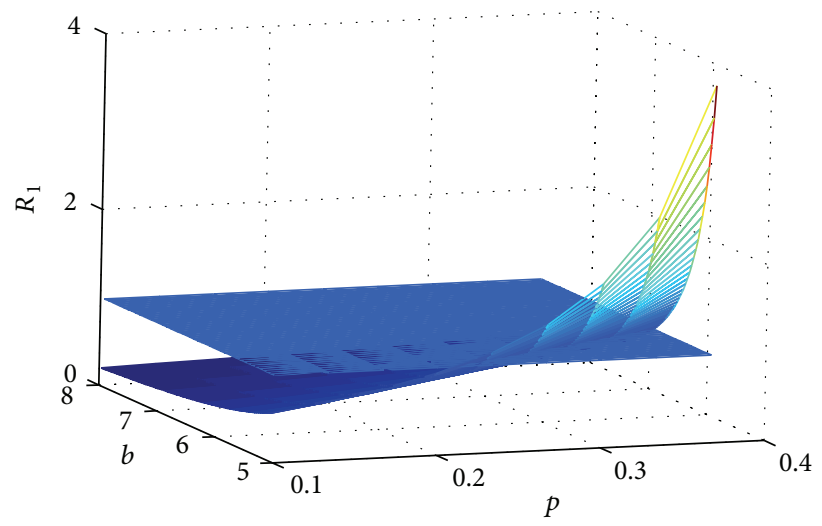

(d)

Figure 3: The influence of some key parameters on the threshold level $R_{1}$; the other parameter values are as follows: $r=3, \omega=0.2, k=$ $0.2, d_{1}=0.1, d_{2}=0.2, d_{3}=0.15$, and $a=5$. (a) $b=7, \tau=0.2, T: 1 \rightarrow 3.8, p: 0.2 \rightarrow 0.6$; (b) $b=7, p=0.25, T: 3.5 \rightarrow 4.5, \tau: 0.1 \rightarrow 0.5$; (c) $b=7, T=4, p: 0.1 \rightarrow 0.5, \tau: 0.1 \rightarrow 0.5$; (d) $\tau=0.2, T=3, b: 5 \rightarrow 8, p: 0.1 \rightarrow 0.4$.

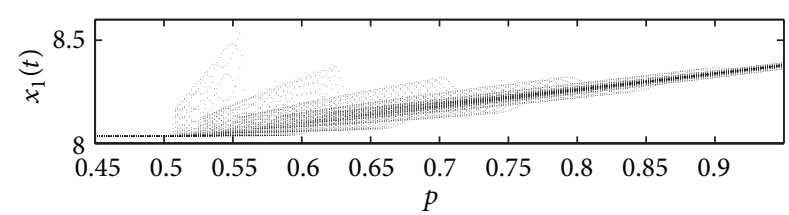

(a)

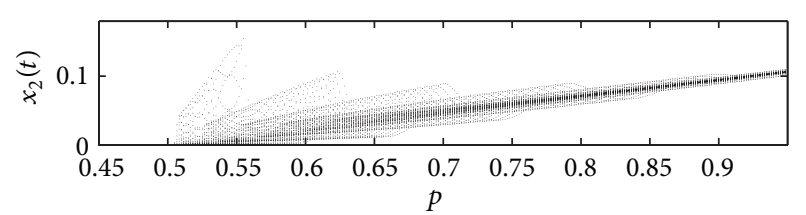

(b)

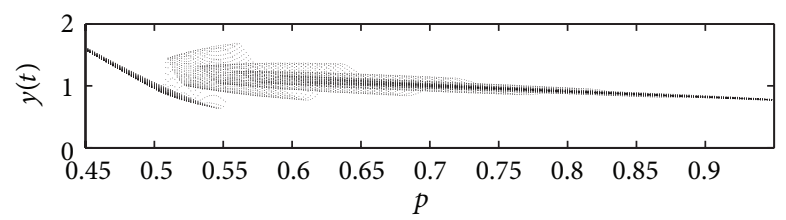

(c)

Figure 4: Complex dynamic properties of system (1) with $r=3, \tau=0.5, \omega=0.2, \mu=4, \beta=4, k=0.2, p_{0}=0.1, d_{1}=0.1, d_{2}=0.2, d_{3}=$ $0.15, a=5, b=7$, and $T=4$, where killing rate $p$ varies from 0.45 to 0.95 .

is, do not spray insecticide, $R_{1}=R_{1}^{*}$, the effect of two control strategies is; if $p \neq 0$, then $R_{1}>R_{1}^{*}$; this result indicates that if natural enemies were born at the same time as spraying insecticide, the side-effects of pesticides on the natural enemy population are very strong. Obviously, part of the newborn natural enemies will be killed and the number of natural enemies will be sharply reduced; the contribution of chemical control for the threshold value $R_{1}$ is stronger than $R_{1}^{*}$. From the practical point of view, we should avoid to spray insecticide when the predators are born. 


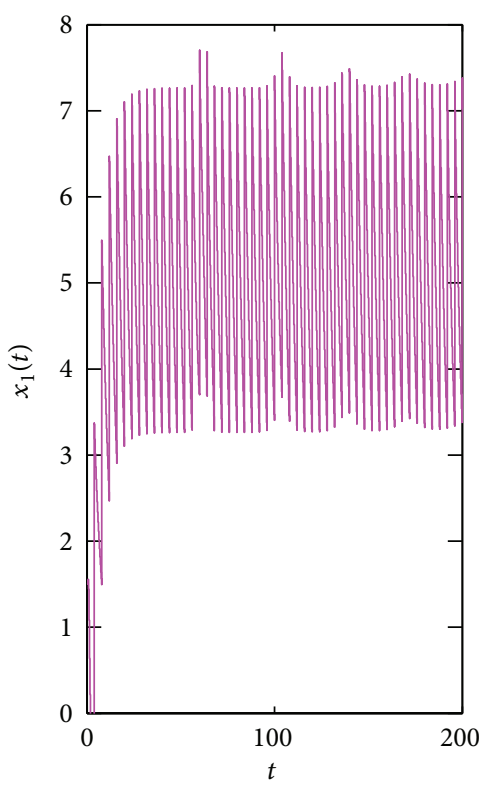

(a)

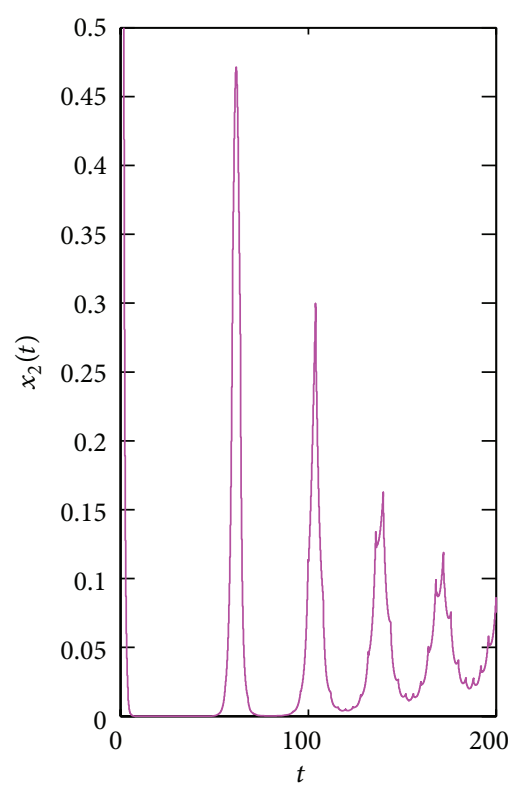

(b)

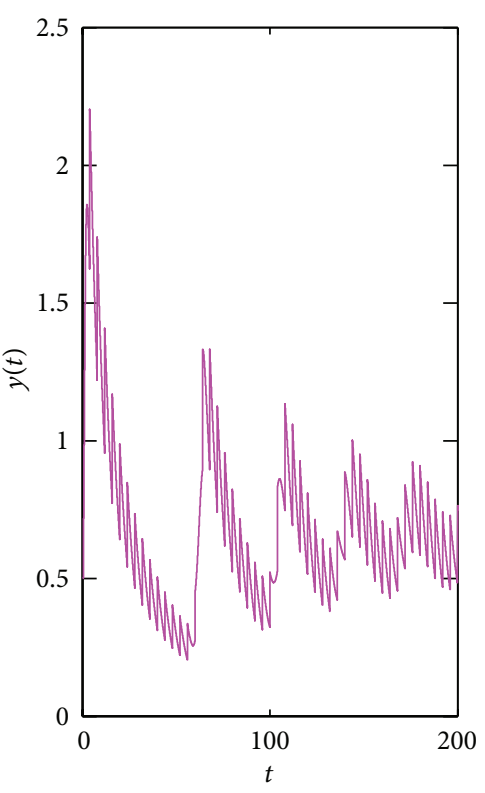

(c)

Figure 5: Time-series of the system (1) with pulse, where killing rate $p=0.7$; other parameters are the same as of those in Figure 4.

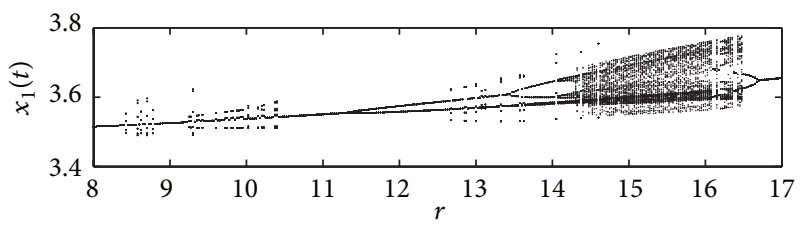

(a)

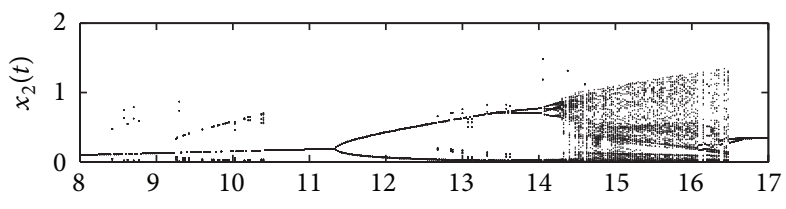

(b)

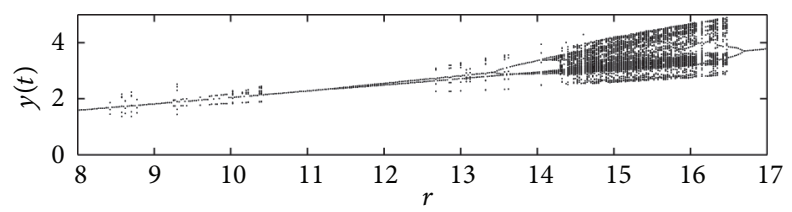

(c)

FIGURE 6: Bifurcation diagrams of system (1) with $\omega=0.2, \beta=4, \mu=4, k=0.2, p_{0}=0.1, p=0.6, d_{1}=0.1, d_{2}=0.2, d_{3}=0.15, \tau=0.05, a=$ $5, b=7$, and $T=4$, where the birth rate $r$ varies from 8 to 17 .

\section{Discussion}

When using integrated pest management as an approach to control insect pests, one must be committed to a long-term strategy. It is well known that pesticides usually act not only on the pest species but also, with even stronger impact, on their natural enemies; then, despite the fact that pesticides kill the target pest, they are simultaneously reducing the population density of natural enemies; the influence of pesticides on natural enemies is even greater than on insect pests. As a result of this indirect effect, treatments can counterintuitively lead to an effective increase of the pest species, even pest breakout again.

In this paper, our idea is to contribute to pest control programs with pesticide applications and with birth pulse in natural enemies and to provide some strategies; that is, spraying pesticides and natural enemies were born at the same time and at a different time. The threshold conditions which guarantee the existence and stability of the mature prey-extinction periodic solution are provided. If 


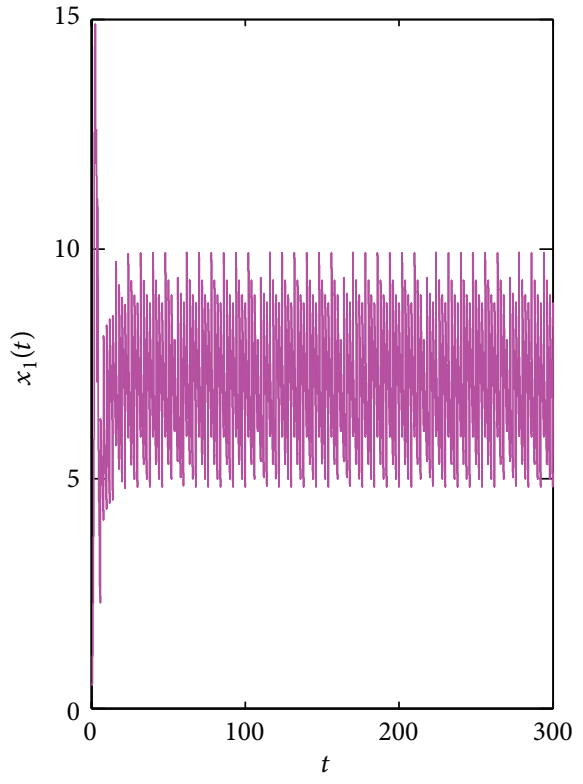

(a)

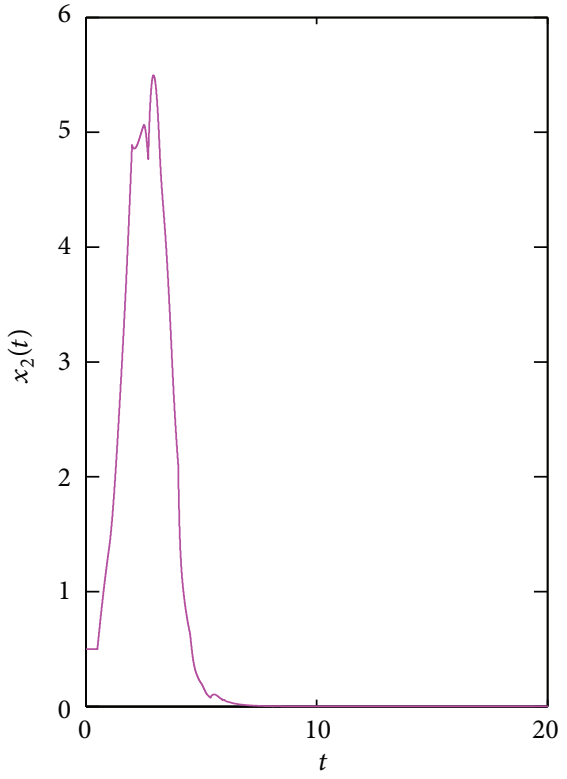

(b)

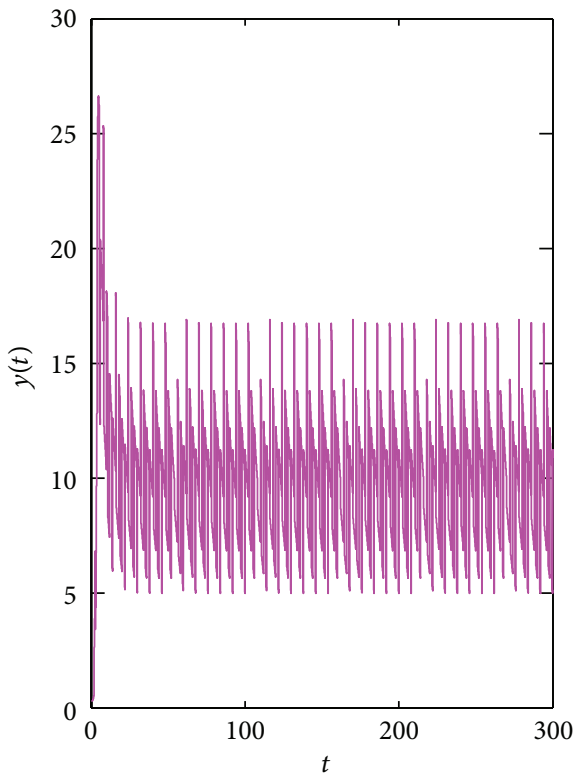

(c)

Figure 7: Dynamical behavior of system (22); the parameter values are as follows: $r=5, \tau=0.5, \omega=0.2, \beta=0.6, k=0.2, d_{1}=0.15, d_{2}=$ $0.2, d_{3}=0.1, p_{0}=0.2, p=0.5, a=3, b=6, T=2, l=0.7$, and $\mu=2$. Here, $R_{1}^{*} \approx 0.8733, p<1-\left(\left(a e^{d_{3} T}\right) /(a+b)\right) \approx 0.7181$.

the integrated control methods cannot completely eradicate the mature prey, the pest population can have outbreaks at different scales. By numerical bifurcation investigations, we found that, when choosing different parameter spaces, two attractors from which the pest population oscillates with different amplitudes can coexist for a wide range of parameters. Moreover, the effects of times of spraying pesticides (or the enemy was born) and control tactics on the threshold conditions were carefully investigated. In particular, the effects of the killing rates of pesticides for pest and natural enemy populations and spraying period on the stability of the mature prey-extinction periodic solutions were discussed. The results imply that the modeling methods described can help the pest control specialist to decide appropriate control strategies and assist management decision-making.

\section{Conflict of Interests}

The authors declare that there is no conflict of interests regarding the publication of this paper. 


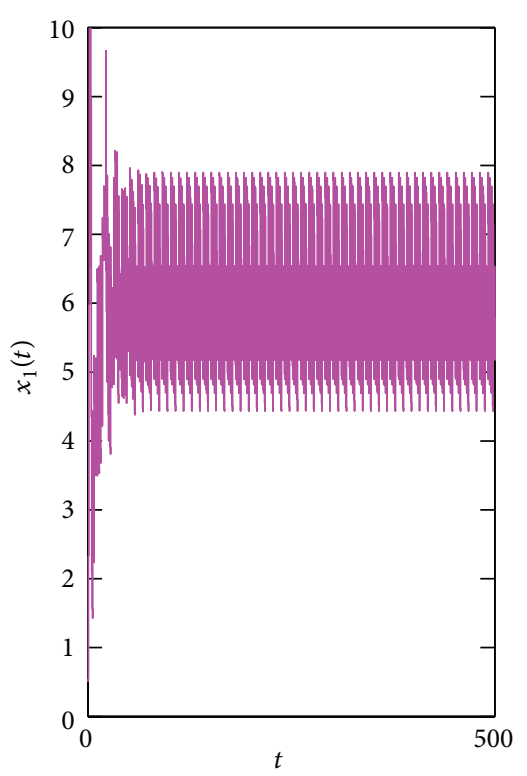

(a)

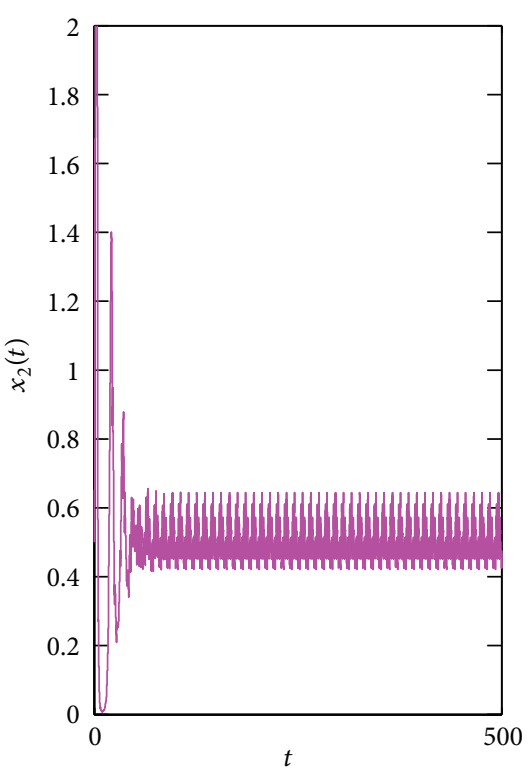

(b)

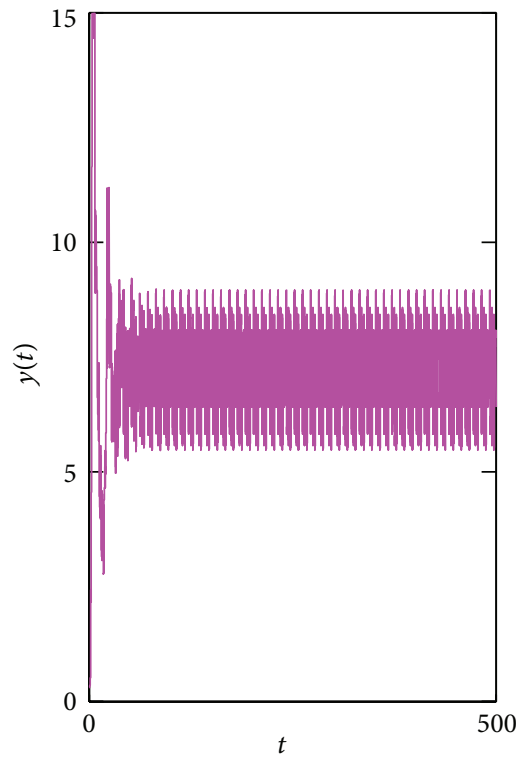

(c)

Figure 8: Dynamical behavior of system (22); the parameter values are as follows: $r=5, \tau=0.5, \omega=0.2, \beta=0.6, k=0.2, d_{1}=0.15, d_{2}=$ $0.2, d_{3}=0.1, p_{0}=0.2, p=0.5, a=1, b=2, T=2, l=0.5$, and $\mu=3$.

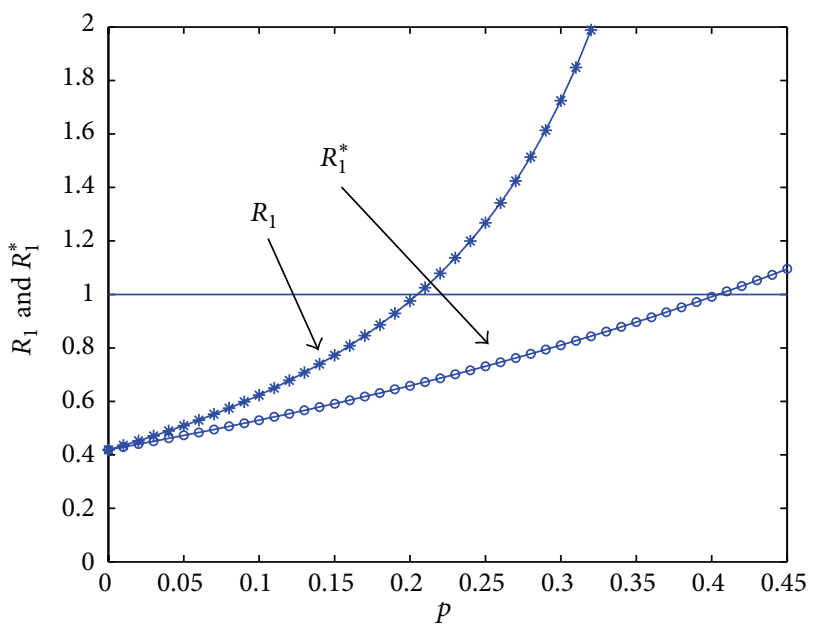

FIGURE 9: Comparing two kinds of control strategy; the parameter values are as follows: $r=4.5, \tau=0.2, \omega=0.2, \beta=2, d_{1}=0.1, d_{3}=$ $0.15, a=2, b=4$, and $T=3$; the killing rate $p$ varies from 0 to 0.45 .

\section{Acknowledgments}

This work is supported by the Key Laboratory of Biologic Resources Protection and Utilization of Hubei Province (PKLHB1302), the Soft Science Research Project of Hubei Province (2012GDA01309), the Key Discipline of Hubei Province-Forestry, and the National Natural Science Foundation of China (11201433).

\section{References}

[1] T. K. Kar and U. K. Pahari, "Modelling and analysis of a preypredator system with stage-structure and harvesting," Nonlinear
Analysis: Real World Applications, vol. 8, no. 2, pp. 601-609, 2007.

[2] H. Wang and W. Wang, "The dynamical complexity of a Ivlev-type prey-predator system with impulsive effect," Chaos, Solitons \& Fractals, vol. 38, no. 4, pp. 1168-1176, 2008.

[3] L. Ling and W. Wang, "Dynamics of a Ivlev-type predatorprey system with constant rate harvesting," Chaos, Solitons and Fractals, vol. 41, no. 4, pp. 2139-2153, 2009.

[4] W. Qin, Z. Liu, and Y. Chen, "Permanence and global stability of positive periodic solutions of a discrete competitive system," Discrete Dynamics in Nature and Society, vol. 2009, Article ID 830537, 13 pages, 2009.

[5] J. Jiao, L. Chen, S. Cai, and L. Wang, "Dynamics of a stagestructured predator-prey model with prey impulsively diffusing between two patches," Nonlinear Analysis: Real World Applications, vol. 11, no. 4, pp. 2748-2756, 2010.

[6] J. Jiao, X. Meng, and L. Chen, "A stage-structured Holling mass defence predator-prey model with impulsive perturbations on predators," Applied Mathematics and Computation, vol. 189, no. 2, pp. 1448-1458, 2007.

[7] W. Wang and L. Chen, "A predator-prey system with stagestructure for predator," Computers \& Mathematics with Applications, vol. 33, no. 8, pp. 83-91, 1997.

[8] C.-Y. Huang, Y.-J. Li, and H.-F. Huo, "The dynamics of a stage-structured predator-prey system with impulsive effect and Holling mass defence," Applied Mathematical Modelling, vol. 36, no. 1, pp. 87-96, 2012.

[9] H. Zhang, L. Chen, and R. Zhu, "Permanence and extinction of a periodic predator-prey delay system with functional response and stage structure for prey," Applied Mathematics and Computation, vol. 184, no. 2, pp. 931-944, 2007.

[10] W.-X. Wang, Y.-B. Zhang, and C.-Z. Liu, "Analysis of a discretetime predator-prey system with Allee effect," Ecological Complexity, vol. 8, no. 1, pp. 81-85, 2011. 
[11] A. J. Terry, "Impulsive adult culling of a tropical pest with a stage-structured life cycle," Nonlinear Analysis: Real World Applications, vol. 11, no. 2, pp. 645-664, 2010.

[12] K. Liu, X. Meng, and L. Chen, "A new stage structured predatorprey Gomportz model with time delay and impulsive perturbations on the prey," Applied Mathematics and Computation, vol. 196, no. 2, pp. 705-719, 2008.

[13] T. K. Kar and A. Ghorai, "Dynamic behaviour of a delayed predator-prey model with harvesting," Applied Mathematics and Computation, vol. 217, no. 22, pp. 9085-9104, 2011.

[14] J. Jiang and Y. Song, "Stability and bifurcation analysis of a delayed Leslie-Gower predator-prey system with nonmonotonic functional response," Abstract and Applied Analysis, vol. 2013, Article ID 152459, 19 pages, 2013.

[15] W. G. Aiello and H. I. Freedman, "A time-delay model of singlespecies growth with stage structure," Mathematical Biosciences, vol. 101, no. 2, pp. 139-153, 1990.

[16] S. Tang and L. Chen, "Density-dependent birth rate, birth pulses and their population dynamic consequences," Journal of Mathematical Biology, vol. 44, no. 2, pp. 185-199, 2002.

[17] Z. Xiang, D. Long, and X. Song, "A delayed Lotka-Volterra model with birth pulse and impulsive effect at different moment on the prey," Applied Mathematics and Computation, vol. 219, no. 20, pp. 10263-10270, 2013.

[18] B. Liu and L. Chen, "The periodic competing Lotka-Volterra model with impulsive effect," Mathematical Medicine and Biology, vol. 21, no. 2, pp. 129-145, 2004.

[19] L. Wang, L. Chen, and J. J. Nieto, "The dynamics of an epidemic model for pest control with impulsive effect," Nonlinear Analysis: Real World Applications, vol. 11, no. 3, pp. 1374-1386, 2010.

[20] X. Wang, Q. Song, and X. Song, "Analysis of a stage structured predator-prey Gompertz model with disturbing pulse and delay," Applied Mathematical Modelling, vol. 33, no. 11, pp. 42314240, 2009.

[21] X. Song and Z. Xiang, "The prey-dependent consumption twoprey one-predator models with stage structure for the predator and impulsive effects," Journal of Theoretical Biology, vol. 242, no. 3, pp. 683-698, 2006.

[22] Y. Xia, J. Cao, and S. S. Cheng, "Multiple periodic solutions of a delayed stage-structured predator-prey model with nonmonotone functional responses," Applied Mathematical Modelling, vol. 31, no. 9, pp. 1947-1959, 2007.

[23] J. Liu, T. Zhang, and J. Lu, "An impulsive controlled ecoepidemic model with disease in the prey," Journal of Applied Mathematics and Computing, vol. 40, no. 1-2, pp. 459-475, 2012.

[24] X. Song and L. Chen, "Optimal harvesting and stability for a two-species competitive system with stage structure," Mathematical Biosciences, vol. 170, no. 2, pp. 173-186, 2001.

[25] V. Lakshmikantham, D. D. Baĭnov, and P. S. Simeonov, Theory of Impulsive Differential Equations, vol. 6 of Series in Modern Applied Mathematics, World Scientific, Singapor, 1989. 


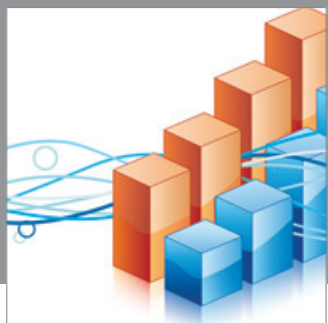

Advances in

Operations Research

mansans

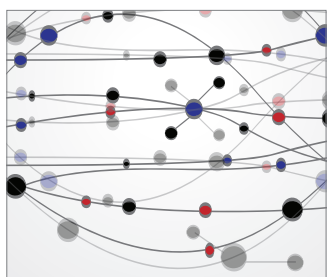

The Scientific World Journal
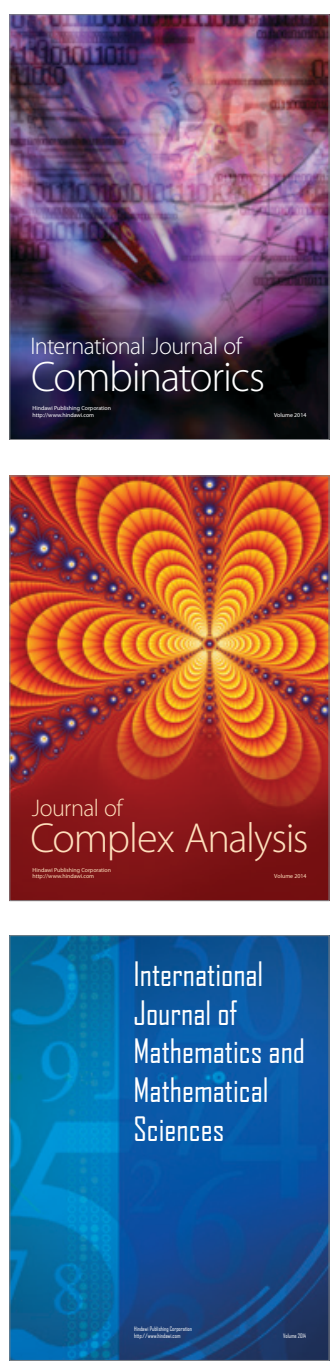
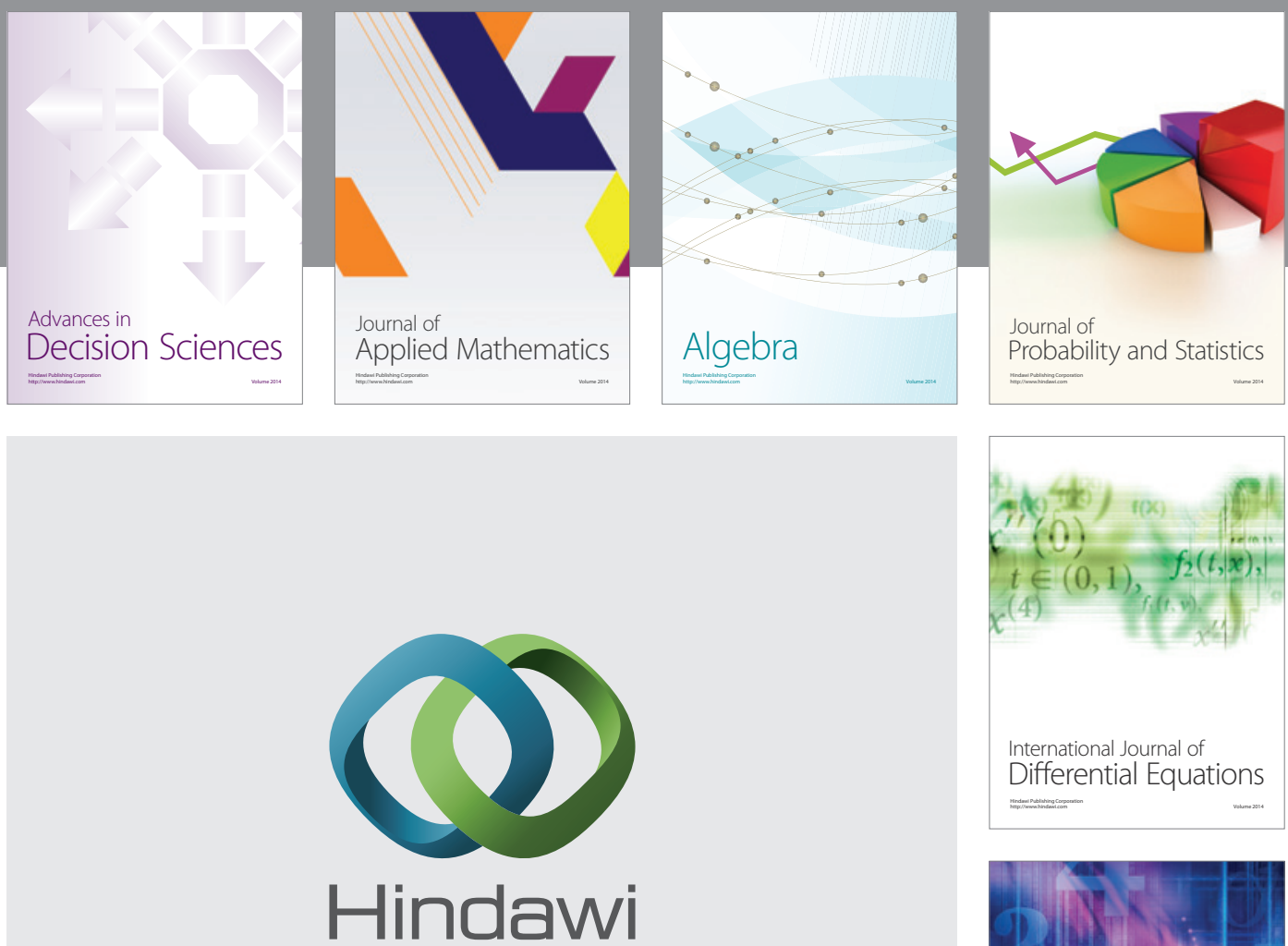

Submit your manuscripts at http://www.hindawi.com
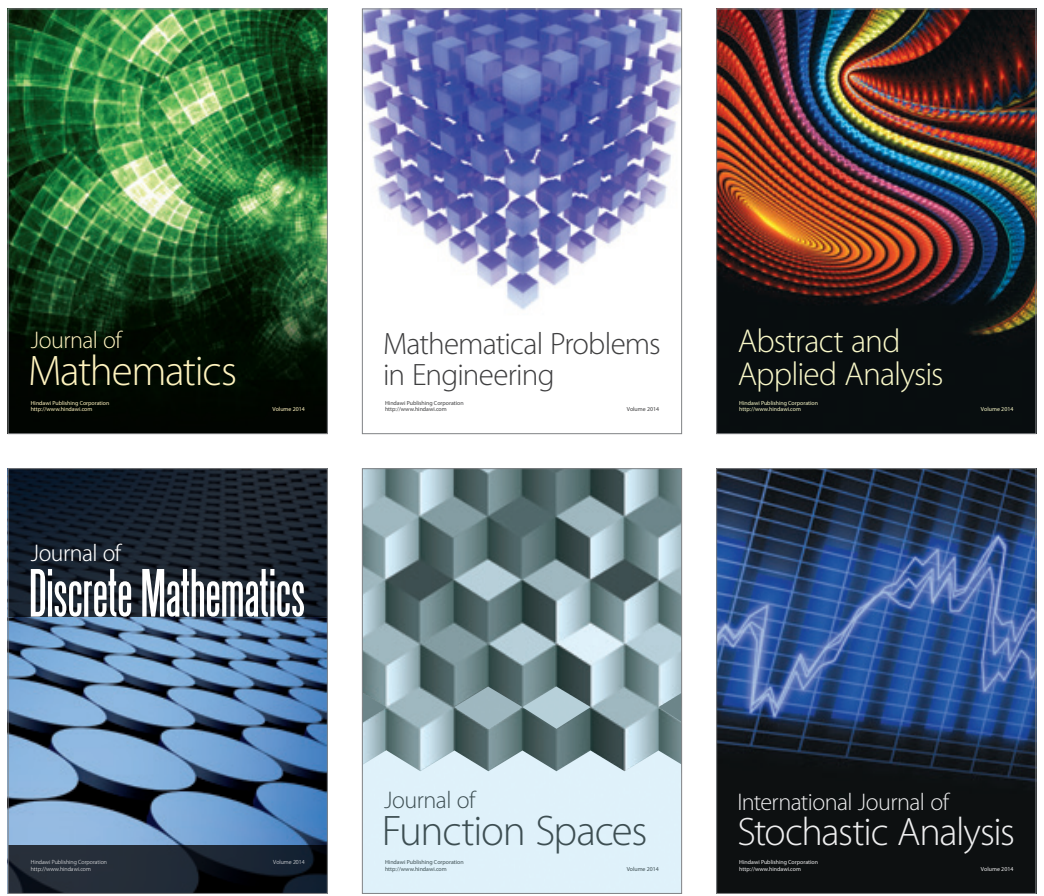

Journal of

Function Spaces

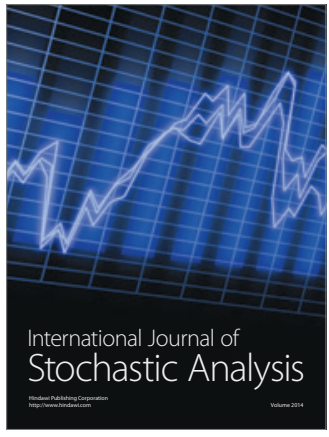

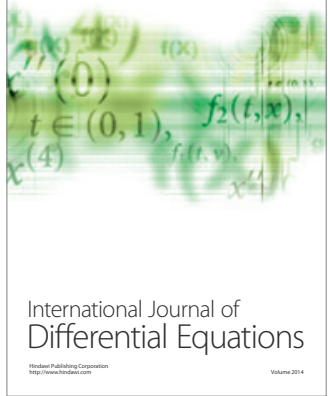
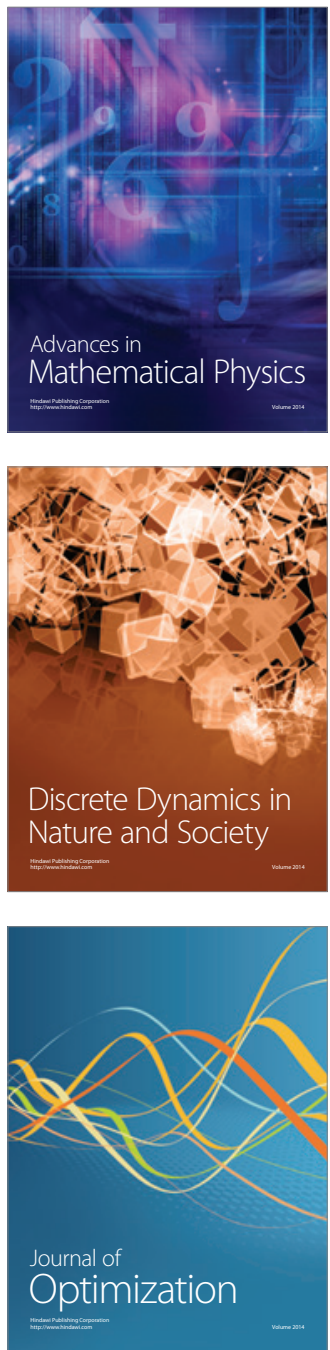